\title{
SOME RECENT ADVANCES IN NONLINEAR DIFFUSION ON NEGATIVELY-CURVED RIEMANNIAN MANIFOLDS: FROM BARRIERS TO SMOOTHING EFFECTS
}

\author{
MATTEO MURATORI
}

\begin{abstract}
In this survey paper we discuss a series of recent results concerning nonnegative solutions to nonlinear diffusion equations of porous-medium type on Cartan-Hadamard manifolds, a special class of negatively-curved Riemannian manifolds that generalize the Euclidean space. We focus on sharp barrier estimates, asymptotic convergence and smoothing effects, describing quantitatively how the curvature behavior at infinity affects the way solutions depart from having a Euclidean-like structure.
\end{abstract}

\section{INTRODUCTION}

The present note is an account of some achievements in the field of nonlinear diffusion equations on Cartan-Hadamard manifolds (i.e. complete and simply connected Riemannian manifolds with nonpositive sectional curvature), which have recently been obtained in a series of papers. The aim is to collect the most important results, in terms of pointwise estimates and asymptotic behavior, concerning nonnegative solutions to the following Cauchy problem for the Porous Medium Equation (PME in the sequel):

$$
\begin{cases}u_{t}=\Delta\left(u^{m}\right) & \text { on } \mathbb{M}^{n} \times(0, \infty), \\ u(\cdot, 0)=u_{0} \geq 0 & \text { on } \mathbb{M}^{n},\end{cases}
$$

where $m>1$ and $\mathbb{M}^{n}$ is a Cartan-Hadamard manifold of dimension $n \geq 2$. The initial datum $u_{0}$ is assumed to be nonnegative and, depending on the specific result we will discuss below, it can be further required to be bounded, compactly supported or globally integrable. Note that the PME is a particular nonlinear version of the Heat Equation, which corresponds to the case $m=1$; it falls within the class of quasilinear parabolic equations of degenerate type, since the diffusion coefficient $m u^{m-1}$ that appears upon writing (1.1) in divergence form vanishes at the free boundary $\partial\{u=0\}$. The case $m \in(0,1)$, which will not be addressed here, gives rise to the so-called Fast Diffusion Equation, where diffusion becomes singular and solutions exhibit quite different behaviors compared to the PME regime. We also mention that one could consider sign-changing initial data (and therefore solutions), in which case $u \mapsto u^{m}$ must be replaced by the monotone power $u \mapsto u|u|^{m-1}$. However, nonnegative solutions enjoy much more interesting properties, hence we will only focus on the latter.

One of the most important features of the PME is the finite speed of propagation, namely the fact that solutions starting from compactly-supported initial data stay with compact support even for positive times, although the support of $u(\cdot, t)$ fills the whole space as $t \rightarrow \infty$. This is in contrast with the heat equation itself, for which the speed of propagation is instantaneously infinite. Clearly such a property makes (1.1) more suitable for modeling several physical phenomena, where real propagation necessarily occurs with finite speed. In the Euclidean setting, that is when $\mathbb{M}^{n}$ is replaced by $\mathbb{R}^{n}$ or by a bounded regular domain of $\mathbb{R}^{n}$ (and therefore (1.1) is completed e.g. with Dirichlet or Neumann conditions), the most complete reference available in the literature for the PME is the monograph [24] by J.L. Vázquez.

A well-established result asserts that, in the whole Euclidean space $\mathbb{M}^{n} \equiv \mathbb{R}^{n}$, nonnegative solutions to (1.1) starting from $L^{1}\left(\mathbb{R}^{n}\right)$ data converge to the fundamental solution $\mathcal{U}_{M}$ having the same mass $M$ as $u_{0}$ (see e.g. [24, Theorem 18.1]):

$$
\lim _{t \rightarrow \infty}\left\|u(\cdot, t)-\mathcal{U}_{M}(\cdot, t)\right\|_{1}=0 \quad \text { and } \quad \lim _{t \rightarrow \infty} t^{\frac{n}{2+n(m-1)}}\left\|u(\cdot, t)-\mathcal{U}_{M}(\cdot, t)\right\|_{\infty}=0 .
$$

In fact $\mathcal{U}_{M}$ is the solution of (1.1) that takes as initial datum a Dirac delta of mass $M$. A similar result was recently proved by Vázquez in [25] for radial solutions on the hyperbolic space $\mathbb{H}^{n}$, which is a particular Cartan-Hadamard manifold of constant sectional curvature equal to -1 . The advantage of working in $\mathbb{R}^{n}$ is that fundamental solutions, also known as Barenblatt solutions after G.I. Barenblatt (we refer to the corresponding literature quoted in [24]), have an explicit profile:

$$
\mathcal{U}_{M}(x, t)=t^{-\frac{n}{2+n(m-1)}}\left(A-B|x|^{2} t^{-\frac{2}{2+n(m-1)}}\right)_{+}^{\frac{1}{m-1}} \quad \forall(x, t) \in \mathbb{R}^{n} \times(0, \infty),
$$


where $B>0$ is a suitable constant depending only on $n, m$ and $A>0$ is a free parameter related to $M$. Unfortunately on Cartan-Hadamard manifolds fundamental solutions are not explicit (not even on $\left.\mathbb{H}^{n}\right)$, although their existence and uniqueness is guaranteed under appropriate curvature bounds [11]. The purpose of the results we present in Section 3, which are based on [12] and [13], is to show how the "approximate profile" of solutions starting from compactly-supported initial data departs from the Euclidean one (given by (1.2) and (1.3)) depending on the behavior of the curvature of $\mathbb{M}^{n}$ at spatial infinity. We will consider curvature bounds of power type, where the power is understood with respect to the geodesic distance from a reference point $o$ (also called the pole of $\mathbb{M}^{n}$ ): see Subsection 2.3. In general it is hopeless to obtain a strong convergence result such as (1.2), hence we will only deal with upper and lower bounds instead, namely pointwise estimates on the solutions from above and below in terms of profiles that take the role of (1.3) on $\mathbb{M}^{n}$. To this end, we exploit classical barrier methods that deeply rely on Laplacian-comparison theorems (see Subsection 2.1). Only a few results will be proved in detail, the purpose being to provide the reader with the basic ideas behind the techniques employed. Different cases, depending on the particular range of curvature considered, are discussed in Subsections $3.1,3.2$ and 3.3 , respectively.

In Euclidean bounded regular domains $\Omega \subset \mathbb{R}^{n}$, with homogeneous Dirichlet boundary conditions, the situation is unlike. Indeed, in [22] it is proved that nonnegative (and nontrivial) solutions converge to a separable profile. More precisely, there holds

$$
\lim _{t \rightarrow \infty}\left\|t^{\frac{1}{m-1}} u(\cdot, t)-v\right\|_{\infty}=0, \quad \text { where } v \geq 0, v \neq \equiv 0, \text { solves } \begin{cases}-\Delta\left(v^{m}\right)=\frac{1}{m-1} v & \text { in } \Omega, \\ v=0 & \text { on } \partial \Omega,\end{cases}
$$

which can be read as uniform convergence of $u$, with rate, to the separable solution $t^{-1 /(m-1)} v$. Surprisingly, we will see in Section 4 that a similar asymptotic behavior takes place on Cartan-Hadamard manifolds with very negative curvature, i.e. having a more-than-quadratic (negative) curvature at infinity. This is one of the main achievements of [13], which will be proved in Subsection 4.2 after a careful analysis of the elliptic equation $-\Delta\left(v^{m}\right)=v /(m-1)$ on $\mathbb{M}^{n}$, carried out in Subsection 4.1.

It is by now well known that there exists a precise connection between the Sobolev inequality, or Gagliardo-Nirenberg inequalities if $n=2$, and the following smoothing effect for solutions to (1.1) in the case $\mathbb{M}^{n} \equiv \mathbb{R}^{n}$ (see for instance the book [23]):

$$
\|u(\cdot, t)\|_{\infty} \leq \mathrm{K} t^{-\frac{n}{2+n(m-1)}}\left\|u_{0}\right\|_{1}^{\frac{2}{2+n(m-1)}} \quad \forall t>0,
$$

where $\mathrm{K}>0$ is a constant depending only on $n, m$. We stress that (1.4) is a direct consequence of the Sobolev inequality, i.e. the proof can be performed by means of a pure functional-analytic approach (we refer e.g. to $[2,9,7]$ ). Note that (1.4) captures exactly the time behavior of the $L^{\infty}$ norm of the Barenblatt solution (1.3), hence the bound is sharp. The aim of Section 5 is to reproduce similar estimates by relying on the validity of new Sobolev-type inequalities for radial functions, that are established in [19] on Cartan-Hadamard manifolds under appropriate curvature bounds. In Subsection 2.2 we will recall some basic facts, such as the validity of the Euclidean Sobolev inequality, on Cartan-Hadamard manifolds. In Subsection 5.1 we will report the main results of [19] and in Subsection 5.2 we will show how the latter can be employed to obtain sharp smoothing effects yielding the correct $L^{\infty}$ time behavior of solutions, that we deduced via the barrier method in Section 3.

Finally, we devote Section 6 to a list of possible future projects that may rise from several questions and problems that are left open after the results of [12], [13] and [19].

\section{Preliminary material}

In the sequel we recall the basic properties of a Cartan-Hadamard manifold that we will exploit below, especially as concerns Laplacian comparison and functional inequalities. Before, let us briefly describe an elementary construction of solutions to (1.1), which is all we need to know to justify the computations of Sections 3 and 4. In order to solve (1.1), one first addresses the following "localized" version:

$$
\begin{cases}\partial_{t} u_{k}=\Delta\left(u_{k}^{m}\right) & \text { in } B_{k} \times(0, \infty), \\ u_{k}=0 & \text { on } \partial B_{k} \times(0, \infty), \\ u_{k}(\cdot, 0)=u_{0} & \text { in } B_{k}\end{cases}
$$

where hereafter by $B_{k}$ we denote the geodesic ball of radius $k$ centered at a pole $o \in \mathbb{M}^{n}$ that is fixed once for all. The solutions to (2.1) can in turn be constructed by an approximation procedure that is typically used in bounded Euclidean domains: the idea is to either raise the datum at the boundary $\partial B_{k}$ by $\varepsilon>0$ or to pick a nondegenerate nonlinearity $u \mapsto \Phi_{\varepsilon}(u)$ that suitably converges to $u \mapsto u^{m}$, and then let 
$\varepsilon \rightarrow 0$. For more details we refer to [24, Chapters 5 and 9] or [3, Section 3], see also Remark 5.5. Finally, one lets $k \rightarrow \infty$ taking advantage of the monotonicity of $\left\{u_{k}\right\}$ (and boundedness if $u_{0}$ is bounded). This procedure gives rise to what is referred to as minimal solution, in the sense that $u:=\lim _{k \rightarrow \infty} u_{k}$ is the smallest solution of (1.1) among nonnegative bounded solutions (see [13]). From here on by "solution" to (1.1) we will implicitly mean the one obtained through the above construction; moreover, for simplicity, properties that a priori may only hold almost everywhere will be stated as if they held everywhere (this is not an issue because the solutions we deal with are continuous [24, Chapter 7]).

2.1. Cartan-Hadamard manifolds, Laplacian comparison and model manifolds. Given $n \geq 2$, a Cartan-Hadamard manifold $\mathbb{M}^{n}$ is an $n$-dimensional Riemannian manifold which is complete, simply connected and has everywhere nonpositive sectional curvature. These assumptions have a very strong topological consequence: any such manifold is diffeomorphic to $\mathbb{R}^{n}$ through the exponential map based at any pole $o \in \mathbb{M}^{n}\left[17\right.$, Theorem 1.10]. In particular one can use polar coordinates: to any $x \in \mathbb{M}^{n} \backslash\{o\}$ it is uniquely associated a couple $(r, \theta) \in(0, \infty) \times \mathbb{S}^{n-1}$, where $\mathbb{S}^{n-1}$ stands for the unit $(n-1)$-dimensional sphere. Note that $r:=\mathrm{d}(x, o)$ is the distance between $x$ and $o$, while $\theta$ is the starting direction of the geodesic connecting $o$ to $x$. Hence, given a smooth function $f \equiv f(r, \theta)$ on $\mathbb{M}^{n}$, the Laplacian (more precisely Laplace-Beltrami) operator applied to $f$ reads [6, Section 3]

$$
\Delta f=\frac{\partial^{2} f}{\partial r^{2}}+\mathrm{m}(r, \theta) \frac{\partial f}{\partial r}+\Delta_{S_{r}} f,
$$

where $\Delta_{S_{r}}$ is the Laplacian operator on the submanifold $S_{r}:=\partial B_{r}$ and $\mathrm{m}(r, \theta)$ is the Laplacian of the distance function $x \equiv(r, \theta) \mapsto r$.

Now we need to introduce the notion of model manifold, namely an $n$-dimensional Riemannian manifold with a pole $o$ whose metric $\mathfrak{g}$ can be written, with respect to the radial coordinates about $o$, as

$$
\mathfrak{g}=d r^{2}+\psi(r)^{2} d \theta^{2},
$$

where $d \theta^{2}$ is the round metric on $\mathbb{S}^{n-1}$ and $\psi:[0, \infty) \rightarrow[0, \infty)$ belongs to the following class:

$$
\mathcal{F}:=\left\{\psi \in C^{\infty}((0, \infty)) \cap C^{1}([0, \infty)): \psi(0)=0, \psi(r)>0 \quad \forall r>0, \psi^{\prime}(0)=1\right\} .
$$

In other words, such manifolds have a spherically-symmetric structure. For a model manifold to be Cartan-Hadamard, it is necessary and sufficient that $\psi$ is in addition convex. Classical examples are the Euclidean space $\mathbb{R}^{n}$, corresponding to $\psi(r) \equiv r$ (sectional curvature $\equiv 0$ ), and the hyperbolic space $\mathbb{H}^{n}$, corresponding to $\psi(r) \equiv \sinh r$ (sectional curvature $\equiv-1$ ). On model manifolds (2.2) reads

$$
\Delta f=\frac{\partial^{2} f}{\partial r^{2}}+(n-1) \frac{\psi^{\prime}}{\psi} \frac{\partial f}{\partial r}+\frac{1}{\psi^{2}} \Delta_{\mathbb{S}^{n-1}} f ;
$$

in particular, in this special case $\mathrm{m}(r, \theta)=(n-1) \psi^{\prime}(r) / \psi(r)$. Classical results allow one to compare the Laplacian of the distance on a Cartan-Hadamard manifold to the Laplacian of the distance on a suitable model manifold, under appropriate curvature bounds (see e.g. [6, Section 15]). More precisely, if

$$
\operatorname{Sect}_{o}(x) \leq-\frac{\psi^{\prime \prime}(r)}{\psi(r)} \quad \forall x \equiv(r, \theta) \in \mathbb{M}^{n} \backslash\{o\}
$$

for some $\psi \in \mathcal{F}$, then

$$
\mathrm{m}(r, \theta) \geq(n-1) \frac{\psi^{\prime}(r)}{\psi(r)} \quad \forall(r, \theta) \in(0, \infty) \times \mathbb{S}^{n-1} ;
$$

similarly, if

$$
\operatorname{Ric}_{o}(x) \geq-(n-1) \frac{\psi^{\prime \prime}(r)}{\psi(r)} \quad \forall x \equiv(r, \theta) \in \mathbb{M}^{n} \backslash\{o\}
$$

for another $\psi \in \mathcal{F}$, then

$$
\mathrm{m}(r, \theta) \leq(n-1) \frac{\psi^{\prime}(r)}{\psi(r)} \quad \forall(r, \theta) \in(0, \infty) \times \mathbb{S}^{n-1} .
$$

Here by $\operatorname{Sect}_{o}(x)$ we denote the sectional curvature at $x \equiv(r, \theta)$ with respect to any plane in the tangent space at $x$ that contains the radial direction (i.e. the radial sectional curvature), and by $\operatorname{Ric}_{o}(x)$ the $\operatorname{Ricci}$ curvature, as a quadratic form, evaluated in the radial direction (i.e. the radial Ricci curvature). In fact (2.4) and (2.6) hold as identities on model manifolds. 
2.2. Functional inequalities and radial functions. On any Cartan-Hadamard manifold $\mathbb{M}^{n}(n \geq 3)$ there hold the Euclidean Sobolev inequality (see for instance [14, Lemma 8.1 and Theorem 8.3])

$$
\|f\|_{L^{2^{*}\left(\mathbb{M}^{n}\right)}} \leq C_{n}\|\nabla f\|_{L^{2}\left(\mathbb{M}^{n}\right)} \quad \forall f \in C_{c}^{1}\left(\mathbb{M}^{n}\right), \quad 2^{*}:=\frac{2 n}{n-2},
$$

where $C_{n}>0$ is a constant depending only on $n$ which, as a consequence of the Cartan-Hadamard conjecture recently settled by [5], can be chosen equal to the optimal constant in $\mathbb{R}^{n}$. For later convenience, the exponent $2^{*}$ in the case $n=2$ is understood as $\infty$. If in addition the sectional curvature is bounded from above by a negative constant $-\mathcal{C}$, then also the Poincaré inequality

$$
\|f\|_{L^{2}\left(\mathbb{M}^{n}\right)} \leq \frac{2}{\sqrt{\mathcal{C}}(n-1)}\|\nabla f\|_{L^{2}\left(\mathbb{M}^{n}\right)} \quad \forall f \in C_{c}^{1}\left(\mathbb{M}^{n}\right)
$$

holds. This is a remarkable result originally due to H.P. McKean [18], which has recently been revisited in [19]. Moreover, the constant of the inequality is "achieved" by the hyperbolic space $\mathbb{H}^{n}$ of sectional curvature $-\mathcal{C}$. It is apparent that both (2.8) and (2.9) can be extended to the whole closure of $C_{c}^{1}\left(\mathbb{M}^{n}\right)$ with respect to the $L^{2}\left(\mathbb{M}^{n}\right)$ norm of the gradient.

Most of our analysis below deals with radial functions. We recall that a radial function is a measurable function $f: \mathbb{M}^{n} \rightarrow \mathbb{R}$ that depends only on $r$, hence $f(x) \equiv f(\mathrm{~d}(x, o)) \equiv f(r)$. In Section 5 we will use the symbol $C_{c: \text { rad }}^{1}\left(\mathbb{M}^{n}\right)$, which stands for the space of radial $C^{1}\left(\mathbb{M}^{n}\right)$ functions with compact support:

$$
C_{c: \operatorname{rad}}^{1}\left(\mathbb{M}^{n}\right):=\left\{f \in C_{c}^{1}\left(\mathbb{M}^{n}\right): f(x) \equiv f(\mathrm{~d}(x, o)) \quad \forall x \in \mathbb{M}^{n}\right\} .
$$

By virtue of (2.2), it is plain that for every $f \in C_{c: r a d}^{1}\left(\mathbb{M}^{n}\right) \cap C^{2}\left(\mathbb{M}^{n}\right)$ there holds

$$
\Delta f=\frac{\partial^{2} f}{\partial r^{2}}+\mathrm{m}(r, \theta) \frac{\partial f}{\partial r} .
$$

2.3. Three significant ranges of curvature. For convenience, we identify three model situations plus two critical cases in which $\operatorname{Sect}_{o}(x)$ (or $\operatorname{Ric}_{o}(x)$ ) has a precise power-type behavior at infinity. That is:

- The quasi-Euclidean range, where $\operatorname{Sect}_{o}(x) \asymp-r^{2 \mu}$ as $r \rightarrow \infty$ for some $\mu<-1$;

- The quasi-Euclidean critical case, where $\operatorname{Sect}_{o}(x) \sim-C r^{-2}$ as $r \rightarrow \infty$ for some $C>0$; this is the sole case where the multiplying constant matters;

- The quasi-hyperbolic range, where $\operatorname{Sect}_{o}(x) \asymp-r^{2 \mu}$ as $r \rightarrow \infty$ for some $\mu \in(-1,1)$; here we further distinguish the left $(\mu \in(-1,0))$, right $(\mu \in(0,1))$ and hyperbolic case $(\mu=0)$;

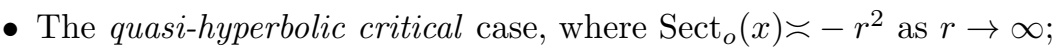

- The super-hyperbolic range, where $\operatorname{Sect}_{o}(x) \asymp-r^{2 \mu}$ as $r \rightarrow \infty$ for some $\mu>1$; this is also referred to as very-negative-curvature range.

Note that by the symbol $\asymp$ we mean that the ratio of two quantities is bounded and bounded away from zero at infinity, while by the symbol $\sim(\approx)$ we mean that it converges to one (to a positive constant).

\section{Sharp POINTWiSE ESTIMATES By THE BARRIER METHOD}

In the following, we will make two types of curvature assumptions:

$$
\operatorname{Sect}_{o}(x) \leq-C r^{2 \mu} \quad \forall x \equiv(r, \theta) \in \mathbb{M}^{n} \backslash B_{R},
$$

or

$$
\operatorname{Ric}_{o}(x) \geq-(n-1) c r^{2 \nu} \quad \forall x \equiv(r, \theta) \in \mathbb{M}^{n} \backslash B_{R},
$$

where $C, c, R>0$ and $\mu, \nu$ are real parameters whose range will be specified below. This is in agreement with the Laplacian-comparison results recalled in Subsection 2.1. Taking advantage of the latter, we will provide explicit pointwise bounds from above and from below for solutions to (1.1) whose initial data are nonnegative, bounded and compactly supported. Depending on the ranges where $\mu$ and $\nu$ vary (see Subsection 2.3) we will obtain qualitatively different estimates, for this reason with split this section in three subsections. Prior to that, we state two important lemmas regarding a significant second-order ODE that is crucially related to the above curvature bounds, whose proofs can be found in [12].

Lemma 3.1 (Lemma 4.1 of [12]). Let $C, R>0$ and $\mu>-1$. Let $\psi$ be the solution of the ODE

$$
\psi^{\prime \prime}(r)=w(r) \psi(r) \quad \forall r>0, \quad \psi(0)=0, \quad \psi^{\prime}(0)=1,
$$

where

$$
w(r)= \begin{cases}0 & \forall r \in[0, R], \\ \frac{C(2 R)^{2 \mu}}{R}(r-R) & \forall r \in(R, 2 R], \\ C r^{2 \mu} & \forall r>2 R .\end{cases}
$$


Then $\psi \in C^{2}([0, \infty)), \psi$ is positive in $(0, \infty)$ with $\lim _{r \rightarrow \infty} \psi(r)=\infty, \psi^{\prime}$ is positive in $[0, \infty)$ and

$$
\lim _{r \rightarrow \infty} \frac{\psi^{\prime}(r)}{r^{\mu} \psi(r)}=\sqrt{C}
$$

Lemma 3.2 (Lemma 4.2 of [12]). Let $c, D, R>0$ and $\nu>-1$. Let $\psi$ be the solution of (3.3), where in the case $\nu \in(-1,0)$ we set

$$
w(r)= \begin{cases}D & \forall r \in[0, R] \\ \frac{c(2 R)^{2 \nu}}{R}(r-R)+\frac{D}{R}(2 R-r) & \forall r \in(R, 2 R] \\ c r^{2 \nu} & \forall r>2 R\end{cases}
$$

whereas in the case $\nu \geq 0$ we set

$$
w(r)=D \vee\left(c r^{2 \nu}\right) \quad \forall r \geq 0
$$

Then $\psi \in C^{2}([0, \infty)), \psi$ is positive in $(0, \infty)$ with $\lim _{r \rightarrow \infty} \psi(r)=\infty, \psi^{\prime}$ is positive in $[0, \infty)$ and the asymptotic identity (3.4) holds with $C=c$ and $\mu=\nu$.

3.1. The quasi-hyperbolic range. We start by considering what we referred to as quasi-hyperbolic range, namely when the power describing the curvature at infinity lies in the interval $(-2,2)$.

Theorem 3.3 (Upper bounds, quasi-hyperbolic, Theorem 3.1 of [12]). Assume that (3.1) is satisfied for some $C, R>0$ and $\mu \in(-1,1)$. Let $u$ be the solution of (1.1) starting from a nonnegative, bounded and compactly-supported initial datum. Then the following pointwise estimate holds:

$$
u(x, t) \leq \frac{\bar{\kappa}}{(t+\bar{\tau})^{\frac{1}{m-1}}}\left(\bar{\gamma}[\log (t+\bar{\tau})]^{\frac{1-\mu}{1+\mu}}-r^{1-\mu}\right)_{+}^{\frac{1}{m-1}} \quad \forall x \equiv(r, \theta) \in \mathbb{M}^{n} \backslash B_{\bar{R}}, \forall t \geq 0,
$$

where $\bar{\kappa}, \bar{\gamma}, \bar{R}, \bar{\tau}$ are positive constants depending only on $n, m, \mu, C, R, u_{0}$. In particular there hold

$$
\|u(\cdot, t)\|_{\infty} \leq \bar{K}\left[\frac{(\log t)^{\frac{1-\mu}{1+\mu}}}{t}\right]^{\frac{1}{m-1}} \quad \text { and } \quad \overline{\mathrm{R}}(t) \leq \bar{\Gamma}(\log t)^{\frac{1}{1+\mu}} \quad \forall t \geq \bar{T},
$$

where $\overline{\mathrm{R}}(t)$ is the radius of the smallest ball containing the support of $u(\cdot, t)$ and $\bar{K}, \bar{\Gamma}, \bar{T}$ are positive constants as above.

Under opposite curvature bounds we are able to prove, up to constants, the reverse of (3.5), thus establishing to some extent the sharpness of our pointwise estimate.

Theorem 3.4 (Lower bounds, quasi-hyperbolic, Theorem 3.2 of [12]). Assume that (3.2) is satisfied for some $c, R>0$ and $\nu \in(-1,1)$. Let $u$ be the solution of (1.1) starting from a nonnegative, bounded, compactly-supported and nontrivial initial datum. Then the following pointwise estimate holds:

$$
u(x, t) \geq \frac{\underline{\kappa}}{(t+\underline{\tau})^{\frac{1}{m-1}}}\left(\underline{\gamma}[\log (t+\underline{\tau})]^{\frac{1-\nu}{1+\nu}}-r^{1-\nu}\right)_{+}^{\frac{1}{m-1}} \quad \forall x \equiv(r, \theta) \in \mathbb{M}^{n} \backslash B_{\underline{R}}, \forall t \geq 0,
$$

where $\underline{\kappa}, \underline{\gamma}, \underline{R}, \underline{\tau}$ are positive constants depending only on $n, m, \nu, c, R, u_{0}$ and on the metric of $\mathbb{M}^{n}$. In particular there hold

$$
\|u(\cdot, t)\|_{\infty} \geq \underline{K}\left[\frac{(\log t)^{\frac{1-\nu}{1+\nu}}}{t}\right]^{\frac{1}{m-1}} \quad \text { and } \quad \underline{\mathrm{R}}(t) \geq \underline{\Gamma}(\log t)^{\frac{1}{1+\nu}} \quad \forall t \geq \underline{T},
$$

where $\underline{\mathrm{R}}(t)$ is the radius of the largest ball contained in the support of $u(\cdot, t)$ and $\underline{K}, \underline{\Gamma}, \underline{T}$ are positive constants as above.

We provide here a (simplified) proof of Theorem 3.3. Full details of the computations can be found in [12]. The rough idea consists of showing that the right-hand side of (3.5) is a supersolution to (1.1), by taking advantage of (2.5) with a choice of the model function $\psi$ driven by Lemma 3.1.

Proof of Theorem 3.3. Let $\psi$ be as in Lemma 3.1 and set the candidate supersolution as follows:

$$
\bar{u}(r, t):=\frac{\kappa}{(t+\tau)^{\frac{1}{m-1}}} \times \begin{cases}\left(\gamma[\log (t+\tau)]^{\frac{1-\mu}{1+\mu}}-r^{1-\mu}\right)_{+}^{\frac{1}{m-1}} & \forall r \geq R_{0}, \\ \left(\gamma[\log (t+\tau)]^{\frac{1-\mu}{1+\mu}}-\frac{1-\mu}{2 R_{0}^{1+\mu}} r^{2}-\frac{1+\mu}{2} R_{0}^{1-\mu}\right)_{+}^{\frac{1}{m-1}} & \forall r \in\left[0, R_{0}\right) .\end{cases}
$$


First of all we show that $\bar{u}$, for a suitable choice of the positive parameters $\kappa, \gamma, R_{0}, \tau$, satisfies

$$
\bar{u}_{t} \geq\left(\bar{u}^{m}\right)_{r r}+(n-1) \frac{\psi^{\prime}}{\psi}\left(\bar{u}^{m}\right)_{r} \quad \text { in }(0, \infty) \times(0, \infty) .
$$

We carry out explicit computations in the region $\left\{r \geq R_{0}\right\}$ only, which is the one where the curvature bound (3.1) plays a major role. For all $r \geq R_{0}$ and $t \geq 0$, taking derivatives we obtain:

$$
\begin{aligned}
(t+\tau)^{\frac{m}{m-1}} \bar{u}_{t}(r, t)= & -\frac{\kappa}{m-1}\left(\gamma[\log (t+\tau)]^{\frac{1-\mu}{1+\mu}}-r^{1-\mu}\right)_{+}^{\frac{1}{m-1}} \\
+ & \frac{\kappa \gamma(1-\mu)}{(1+\mu)(m-1)[\log (t+\tau)]^{\frac{2 \mu}{1+\mu}}}\left(\gamma[\log (t+\tau)]^{\frac{1-\mu}{1+\mu}}-r^{1-\mu}\right)_{+}^{\frac{2-m}{m-1}} \\
(t+\tau)^{\frac{m}{m-1}}\left(\bar{u}^{m}\right)_{r}(r, t)= & -\frac{m \kappa^{m}(1-\mu)}{(m-1) r^{\mu}}\left(\gamma[\log (t+\tau)]^{\frac{1-\mu}{1+\mu}}-r^{1-\mu}\right)_{+}^{\frac{1}{m-1}} \\
(t+\tau)^{\frac{m}{m-1}}\left(\bar{u}^{m}\right)_{r r}(r, t)= & \frac{m \kappa^{m} \mu(1-\mu)}{(m-1) r^{1+\mu}\left(\gamma[\log (t+\tau)]^{\frac{1-\mu}{1+\mu}}-r^{1-\mu}\right)_{+}^{\frac{1}{m-1}}} \\
& +\frac{m \kappa^{m}(1-\mu)^{2}}{(m-1)^{2} r^{2 \mu}}\left(\gamma[\log (t+\tau)]^{\frac{1-\mu}{1+\mu}}-r^{1-\mu}\right)_{+}^{\frac{2-m}{m-1}}
\end{aligned}
$$

Note that, when $m>2$, the right-hand side of the time derivative and of the second spatial derivative is singular at the boundary of the support of $\bar{u}$; however, this is not an issue up to interpreting (3.10) in weak sense (see also Remark 3.5). In order for $\bar{u}$ to be a supersolution in $\left\{r \geq R_{0}\right\}$, we start by picking $R_{0}$ (depending on $n, \mu, C, R$ ) so large that

$$
(n-1) \frac{\psi^{\prime}(r)}{\psi(r) r^{\mu}}-\frac{\mu}{r^{1+\mu}} \geq(n-1) \sqrt{C / 2} \quad \forall r \geq R_{0} .
$$

This is possible since $\mu>-1$ by assumption and Lemma 3.1 ensures the validity of (3.4). Thanks to (3.11) and the above explicit expressions of the derivatives, it is readily seen that a sufficient condition for $(3.10)$ to hold in the region $\left[R_{0}, \infty\right) \times(0, \infty)$ is the following:

$$
\begin{aligned}
& {\left[m(1-\mu)(n-1) \sqrt{C / 2} \kappa^{m-1}-1\right]\left(\gamma[\log (t+\tau)]^{\frac{1-\mu}{1+\mu}}-r^{1-\mu}\right)_{+}^{\frac{1}{m-1}} } \\
+ & \frac{\gamma(1-\mu)}{(1+\mu)[\log (t+\tau)]^{\frac{2 \mu}{1+\mu}}}\left(\gamma[\log (t+\tau)]^{\frac{1-\mu}{1+\mu}}-r^{1-\mu}\right)_{+}^{\frac{2-m}{m-1}} \\
\geq & \frac{m \kappa^{m-1}(1-\mu)^{2}}{(m-1) r^{2 \mu}}\left(\gamma[\log (t+\tau)]^{\frac{1-\mu}{1+\mu}}-r^{1-\mu}\right)_{+}^{\frac{2-m}{m-1}} .
\end{aligned}
$$

Upon choosing $\kappa, \gamma, \tau$ sufficiently large, inequality (3.12) can be fulfilled. Indeed, given $\mathrm{M} \geq 1$, let us set

$$
\kappa=\left[\frac{2 \mathrm{M}}{m(1-\mu)(n-1) \sqrt{C / 2}}\right]^{\frac{1}{m-1}}
$$

so that (3.12) reads

$$
\begin{aligned}
& (2 \mathrm{M}-1)\left(\gamma[\log (t+\tau)]^{\frac{1-\mu}{1+\mu}}-r^{1-\mu}\right)_{+}^{\frac{1}{m-1}}+\frac{\gamma(1-\mu)}{(1+\mu)[\log (t+\tau)]^{\frac{2 \mu}{1+\mu}}}\left(\gamma[\log (t+\tau)]^{\frac{1-\mu}{1+\mu}}-r^{1-\mu}\right)_{+}^{\frac{2-m}{m-1}} \\
\geq & \frac{2 \mathrm{M}(1-\mu)}{(m-1)(n-1) \sqrt{C / 2} r^{2 \mu}}\left(\gamma[\log (t+\tau)]^{\frac{1-\mu}{1+\mu}}-r^{1-\mu}\right)_{+}^{\frac{2-m}{m-1}} .
\end{aligned}
$$

In the space-time region

$$
R_{0}^{1-\mu} \leq r^{1-\mu}<\gamma[\log (t+\tau)]^{\frac{1-\mu}{1+\mu}},
$$

namely where the argument of the positive part is nonzero, one can rewrite (3.14) as

$$
\gamma(2 \mathrm{M}-1)[\log (t+\tau)]^{\frac{1-\mu}{1+\mu}}+\frac{\gamma(1-\mu)}{(1+\mu)[\log (t+\tau)]^{\frac{2 \mu}{1+\mu}}} \geq \frac{2 \mathrm{M}(1-\mu)}{(m-1)(n-1) \sqrt{C / 2} r^{2 \mu}}+(2 \mathrm{M}-1) r^{1-\mu} .
$$

With no loss of generality, we can and will assume that $\gamma \geq 1$ and $\log \tau>R_{0}^{1+\mu}$ : this makes sure that (3.15) is never empty. An elementary computation shows that if

$$
R_{0}^{1+\mu} \geq \frac{4 \mu}{(m-1)(n-1) \sqrt{C / 2}}
$$


then the right-hand side of (3.16) is monotone increasing for all $r \geq R_{0}$. As a consequence, recalling assumption (3.15), for the validity of (3.16) it is enough to require that the latter is satisfied at

$$
r=\gamma^{\frac{1}{1-\mu}}[\log (t+\tau)]^{\frac{1}{1+\mu}}
$$

which is equivalent to the condition

$$
\gamma \geq\left[\frac{2 \mathrm{M}(1+\mu)}{(m-1)(n-1) \sqrt{C / 2}}\right]^{\frac{1-\mu}{1+\mu}} .
$$

We have therefore shown that $\bar{u}$ complies with (3.10) in the region (3.15) provided $\kappa$ is chosen as in (3.13) for some $\mathrm{M} \geq 1, \gamma$ is larger than 1 and fulfills (3.19), $\log \tau>R_{0}^{1+\mu}$ and $R_{0}$ satisfies (3.17). As discussed previously, we can safely extend the validity of (3.10) to the whole region $\left\{r \geq R_{0}\right\}$ in weak sense.

By construction, the function $\bar{u}$ is $C^{1}$ through $\left\{r=R_{0}\right\}$ independently of the choice of the parameters, so that the derivative with respect to $r$ has no jumps and therefore, in order to prove (3.10) globally (in weak sense), we are only left with handling the region $\left\{r \in\left(0, R_{0}\right)\right\}$. Here we do not provide full details and only mention that computations similar to those carried out above lead to the following constraints:

$$
\mathrm{M} \geq \frac{(n-1) \sqrt{C / 2} R_{0}^{1+\mu}}{a+1} \quad \text { and } \quad \gamma \geq 1+\frac{2 \mathrm{M}(1-\mu)}{(m-1)\left[2 \mathrm{M}(a+1)-(n-1) \sqrt{C / 2} R_{0}^{1+\mu}\right]},
$$

where $\mathrm{M} \geq 1$ is still the constant appearing in (3.13) and

$$
a:=(n-1) \inf _{r \in\left(0, R_{0}\right)} \frac{\psi^{\prime}(r) r}{\psi(r)},
$$

i.e. $a$ is a suitable positive constant depending only on $n, \mu, C, R, R_{0}$ (recall the properties of $\psi$ guaranteed by Lemma 3.1). In conclusion, upon collecting the results obtained in the two spatial regions $\left\{r \geq R_{0}\right\}$ and $\left\{r \in\left(0, R_{0}\right)\right\}$, along with (3.13), we can finally assert that $\bar{u}$ satisfies (3.10) provided:

$$
\begin{gathered}
R_{0} \geq\left[\frac{4}{(m-1)(n-1) \sqrt{C / 2}}\right]^{\frac{1}{1+\mu}}, \quad \tau>e^{R_{0}^{1+\mu}}, \\
\kappa \geq\left[\frac{2}{m(1-\mu)}\right]^{\frac{1}{m-1}}\left[\frac{1}{(n-1) \sqrt{C / 2}} \vee \frac{R_{0}^{1+\mu}}{a+1}\right]^{\frac{1}{m-1}}, \\
\gamma \geq\left[\frac{m(1+\mu)(1-\mu) \kappa^{m-1}}{m-1}\right]^{\frac{1-\mu}{1+\mu}} \vee\left(1+\frac{m(1-\mu)^{2} \kappa^{m-1}}{(m-1)\left[m(1-\mu)(1+a) \kappa^{m-1}-R_{0}^{1+\mu}\right]}\right) .
\end{gathered}
$$

Regardless of the explicit expression of the bounds, it is important to observe that the latter describe a feasible range of parameters. This is easily seen upon choosing them according to the above order: first one picks $R_{0}$ depending on $n, m, \mu, C$, then $\tau$ depending on $\mu, R_{0}$, then $\kappa$ depending on $n, m, \mu, C, R_{0}, a$ and finally $\gamma$ depending on $n, m, \mu, R_{0}, a, \kappa$.

In order to prove the thesis, we need to show how the barrier $\bar{u}$ can be exploited. The curvature assumption (3.1) plus the fact that $\mathbb{M}^{n}$ is Cartan-Hadamard, namely the sectional curvature is everywhere nonpositive (in particular for $r \leq 2 R$ ), yield

$$
\operatorname{Sect}_{o}(x) \leq-\frac{\psi^{\prime \prime}(r)}{\psi(r)} \quad \forall x \equiv(r, \theta) \in \mathbb{M}^{n} \backslash\{o\},
$$

still with the same $\psi$ as in Lemma 3.1. Hence Laplacian comparison ensures that (2.5) holds. Because $\bar{u}$ is nonincreasing in $r$ and complies with (3.10) (subject to (3.20)-(3.22)), recalling (2.10) we have:

$$
\bar{u}_{t} \geq\left(\bar{u}^{m}\right)_{r r}+(n-1) \frac{\psi^{\prime}}{\psi}\left(\bar{u}^{m}\right)_{r} \geq\left(\bar{u}^{m}\right)_{r r}+\mathrm{m}(r, \theta)\left(\bar{u}^{m}\right)_{r}=\Delta\left(\bar{u}^{m}\right) \quad \text { on } \mathbb{M}^{n} \times(0, \infty),
$$

where it is understood that $\bar{u}(x, t) \equiv \bar{u}(r, t)$ by means of the identity $r=\mathrm{d}(x, o)$. Note that through the pole $o$ there are no issues passing from (3.10) to (3.23), since by construction the radial derivative of $\bar{u}^{m}$ vanishes at $r=0$. Hence we have established that $\bar{u}$ is a supersolution to the differential equation in (1.1). We are left with checking the initial condition, namely

$$
\bar{u}(\cdot, 0) \geq u_{0} \quad \text { on } \mathbb{M}^{n} .
$$

Let us assume that $R_{0}$ is so large that

$$
B_{R_{0}} \supset \operatorname{supp} u_{0}
$$


then it is readily seen that a sufficient condition for (3.24) to hold is

$$
\kappa \geq \tau^{\frac{1}{m-1}}\left\|u_{0}\right\|_{\infty} \quad \text { and } \quad \gamma \geq \frac{1+R_{0}^{1-\mu}}{[\log \tau]^{\frac{1-\mu}{1+\mu}}} .
$$

As a result, we can finally assert that $\bar{u}$ is a supersolution to the Cauchy problem (1.1) provided $\kappa, \gamma, R_{0}, \tau$ comply with the compatible conditions (3.20)-(3.22) and (3.25)-(3.26), so that by the comparison principle (see Remark 3.5) there follows $u \leq \bar{u}$ on $\mathbb{M}^{n} \times[0, \infty)$. Hence (3.5) is proved upon replacing $\kappa, \gamma, R_{0}, \tau$ with $\bar{\kappa}, \bar{\gamma}, \bar{R}, \bar{\tau}$, respectively (we have relabeled parameters only to lighten notations along the proof). Also (3.6) is a direct consequence of the inequality $u \leq \bar{u}$, along with the fact that $\bar{u}(\cdot, t)$ is supported in the ball of radius given by (3.18) and attains the maximum at $r=0$.

Theorem 3.4 follows by a similar barrier approach, which takes advantage of (2.7) in place of (2.5).

Proof of Theorem 3.4. We will only give a sketch, emphasizing the differences with respect to the proof of Theorem 3.3. We consider a candidate subsolution, which we label $\underline{u}$, exactly of the same form as $\bar{u}$ in (3.9), with $\mu$ replaced by $\nu$. Here we aim at showing that such a function satisfies

$$
\underline{u}_{t} \leq\left(\underline{u}^{m}\right)_{r r}+(n-1) \frac{\psi^{\prime}}{\psi}\left(\underline{u}^{m}\right)_{r} \quad \text { in }(0, \infty) \times(0, \infty)
$$

where $\psi$ is the model function of Lemma 3.2 with

$$
D=\max \left\{\frac{1}{n-1} \sup _{x \in B_{R}}-\operatorname{Ric}_{o}(x), c R^{2 \nu}\right\},
$$

up to choosing the parameters appropriately. More precisely, computations very similar to those performed above ensure that $\underline{u}$ complies with (3.27) provided:

$$
\begin{gathered}
R_{0} \geq[(m-1)(n-1) \sqrt{c / 2}]^{-\frac{1}{1+\nu}} \\
0<\kappa^{m-1} \leq \frac{1}{m(1-\nu)}\left(\frac{1}{2(n-1) \sqrt{2 c}} \wedge \frac{R_{0}^{1+\nu}}{4(b+1)} \wedge \frac{(m-1) R_{0}^{1+\nu}}{[2+(m-1)(b+1)]}\right) \\
0<\gamma \leq\left[\frac{m(1+\nu)(1-\nu) \kappa^{m-1}}{m-1}\right]^{\frac{1-\nu}{1+\nu}}, \\
\log \tau \geq \frac{R_{0}^{1+\nu}}{\gamma^{\frac{1+\nu}{1-\nu}}}\left(\frac{4(m-1) R_{0}^{1+\nu}-4 m(1-\nu)[(m-1)(b+1)+1-\nu] \kappa^{m-1}}{(3+\nu)(m-1) R_{0}^{1+\nu}-4 m(m-1)(1-\nu)(b+1) \kappa^{m-1}}\right)^{\frac{1+\nu}{1-\nu}}
\end{gathered}
$$

where $b$ is defined as

$$
b:=(n-1) \sup _{r \in\left(0, R_{0}\right)} \frac{\psi^{\prime}(r) r}{\psi(r)} .
$$

It is easy to check that, in view of the curvature assumption (3.2), there holds

$$
\operatorname{Ric}_{o}(x) \geq-(n-1) \frac{\psi^{\prime \prime}(r)}{\psi(r)} \quad \forall x \equiv(r, \theta) \in \mathbb{M}^{n} \backslash\{o\} .
$$

We are therefore in position to exploit Laplacian comparison through (2.7), which combined with (3.27) and the fact that $\underline{u}(x, t) \equiv \underline{u}(r, t)$ is a nonincreasing function of $r$ yields

$$
\underline{u}_{t} \leq\left(\underline{u}^{m}\right)_{r r}+(n-1) \frac{\psi^{\prime}}{\psi}\left(\underline{u}^{m}\right)_{r} \leq\left(\underline{u}^{m}\right)_{r r}+\mathrm{m}(r, \theta)\left(\underline{u}^{m}\right)_{r}=\Delta\left(\underline{u}^{m}\right) \quad \text { on } \mathbb{M}^{n} \times(0, \infty) .
$$

We have thus established that $\underline{u}$, of the form (3.9), is a subsolution to the differential equation in (1.1) under the validity of $(3.28)-(3.31)$. It remains to be checked that

$$
\underline{u}(\cdot, 0) \leq u_{0} \quad \text { on } \mathbb{M}^{n} .
$$

First of all note that, by virtue of (3.29), condition (3.31) is surely satisfied if one imposes

$$
\log \tau=\frac{R_{0}^{1+\nu}}{\gamma^{\frac{1+\nu}{1-\nu}}} H^{\frac{1+\nu}{1-\nu}}
$$

for a suitable constant $H>1$ that depends only on $n, m, \nu, c, R_{0}, b$ (in particular independent of $\kappa$ ). For instance, one can take $H$ as the supremum of the factor between large brackets in the right-hand side of (3.31) as $\kappa$ ranges in the interval (3.29). Thanks to the local positivity properties of the PME 
(see Remark 3.6) we can assert that there exist a time $t_{0}>0$ and a constant $I_{0}>0$, depending on $n, m, R_{0}, H, u_{0}$ and on the local metric structure of $\mathbb{M}^{n}$, such that

$$
\inf _{x \in B_{\mathcal{R}}} u\left(x, t_{0}\right) \geq I_{0}, \quad \text { where } \mathcal{R}:=H^{\frac{1}{1-\nu}} R_{0} .
$$

Recalling that the maximum of $\underline{u}(\cdot, 0)$ is attained at $r=0$ and $\tau>1$ under (3.33), it is then straightforward to show that $(3.32)$, with $u_{0}$ replaced by $u\left(\cdot, t_{0}\right)$, holds provided

$$
\kappa \leq \frac{I_{0}}{\left(H-\frac{1+\nu}{2}\right)^{\frac{1}{m-1}} R_{0}^{\frac{1-\nu}{m-1}}} .
$$

In conclusion, by the comparison principle applied on $\mathbb{M}^{n} \times\left(t_{0}, \infty\right)$, we deduce that if $\kappa, \gamma, R_{0}, \tau$ comply with (3.28), (3.29), (3.34), (3.30) and (3.33), there follows

$$
u(x, t) \geq \underline{u}\left(x, t-t_{0}\right) \quad \text { on } \mathbb{M}^{n} \times\left[t_{0}, \infty\right) .
$$

Upon observing the trivial inequality

$$
\log \left(t-t_{0}+\tau\right) \geq \frac{1}{2} \log \left(t+t_{0}+\tau\right) \quad \forall t \geq t_{0}+\frac{1+\sqrt{8 t_{0}+1}}{2}-\tau=: \tilde{t}_{0}-\tau,
$$

by setting $\underline{\tau}=t_{0}+\tau, \underline{\kappa}=\kappa$ and $\underline{\gamma}=\gamma / 2^{\frac{1-\nu}{1+\nu}}$, from (3.35) we can infer in particular that

$$
u(x, t) \geq \frac{\underline{\kappa}}{(t+\underline{\tau})^{\frac{1}{m-1}}}\left(\underline{\gamma}[\log (t+\underline{\tau})]^{\frac{1-\nu}{1+\nu}}-r^{1-\nu}\right)_{+}^{\frac{1}{m-1}} \quad \forall x \equiv(r, \theta) \in \mathbb{M}^{n} \backslash B_{R_{0}}, \forall t \geq \tilde{t}_{0} .
$$

Finally, in order to pass from (3.36) to (3.7) it is enough to pick

$$
\underline{R}=R_{0} \vee\left(\underline{\gamma}^{\frac{1}{1-\nu}}\left[\log \left(\tilde{t}_{0}+\underline{\tau}\right)\right]^{\frac{1}{1+\nu}}\right),
$$

thus ensuring that the argument of the positive part is nonpositive for all $t \leq \tilde{t}_{0}$ and $r \geq \underline{R}$. The validity of (3.8) then follows plainly as in the proof of Theorem 3.3.

Remark 3.5 (On the comparison principle). In the proofs of the above results, we implicitly used a comparison principle for super-, sub- and solutions to (1.1). A rigorous justification of the latter requires working with the approximate solutions $u_{k}$ to $(2.1)$, where $\partial B_{k}$ plays no role due to the homogeneous Dirichlet conditions. The "standard" method consists of multiplying the differential inequality $\partial_{t}\left(\bar{u}-u_{k}\right) \geq \Delta\left(\bar{u}^{m}-u_{k}^{m}\right)$ by $\operatorname{sign}\left(u_{k}^{m}-\bar{u}^{m}\right)_{+}$and integrating, upon further approximating the sign function. For more details we refer to [24, Theorem 8.10]. The inequality may only hold in weak sense, but in order for the procedure to be successful it is essential that both $\bar{u}$ and $u_{k}$ are strong, i.e. they possess an $L^{1}$ time derivative, which is the case thanks to the explicit profile of $\bar{u}$ and [24, Corollary 8.3] (the fact that the setting there is Euclidean is irrelevant). Similar observations hold for $\underline{u}$.

Remark 3.6 (Expansion of positivity for the PME). A crucial property of nonnegative solutions to (1.1), that we exploit in the proof of Theorem 3.4, is the expansion of positivity, namely the fact that for every $R_{0}>0$ there exists a time $T_{0}$ such that the infimum of $u(\cdot, t)$ in $B_{R_{0}}$ is strictly positive for all $t \geq T_{0}$, provided $u_{0} \not \equiv 0$. We refer to [24, Chapter 14] for a thorough analysis in the Euclidean framework; as a key observation we point out that (1.1), written in local coordinates, becomes a weighted Euclidean PME with (locally) uniformly elliptic coefficients.

3.2. The super-hyperbolic range. Below we state the results of [13] regarding sharp pointwise estimates for (1.1) in the super-hyperbolic range, i.e. when the curvature is more than quadratic at infinity.

Theorem 3.7 (Upper bounds, super-hyperbolic, Theorem 2.8 of [13]). Assume that (3.1) is satisfied for some $C, R>0$ and $\mu>1$. Let $u$ be the solution of (1.1) starting from a nonnegative, bounded and compactly-supported initial datum. Then the following pointwise estimate holds:

$$
u(x, t) \leq \frac{\bar{\kappa}}{(t+\bar{\tau})^{\frac{1}{m-1}}}\left(\frac{1}{(r+\bar{R})^{\mu-1}}-\frac{\bar{\gamma}}{[\log (t+\bar{\tau})]^{\frac{\mu-1}{\mu+1}}}\right)_{+}^{\frac{1}{m-1}} \quad \forall x \equiv(r, \theta) \in \mathbb{M}^{n}, \forall t \geq 0,
$$

where $\bar{\kappa}, \bar{\gamma}, \bar{R}, \bar{\tau}$ are positive constants depending only on $n, m, \mu, C, R, u_{0}$. In particular there hold

$$
\|u(\cdot, t)\|_{\infty} \leq \frac{\bar{K}}{t^{\frac{1}{m-1}}} \quad \text { and } \quad \overline{\mathrm{R}}(t) \leq \bar{\Gamma}(\log t)^{\frac{1}{1+\mu}} \quad \forall t \geq \bar{T},
$$

where $\overline{\mathrm{R}}(t)$ is the radius of the smallest ball containing the support of $u(\cdot, t)$ and $\bar{K}, \bar{\Gamma}, \bar{T}$ are positive constants as above. 
Theorem 3.8 (Lower bounds, super-hyperbolic, Theorem 2.9 of [13]). Assume that (3.2) is satisfied for some $c, R>0$ and $\nu>1$. Let $u$ be the solution of (1.1) starting from a nonnegative, bounded, compactly-supported and nontrivial initial datum. Then the following pointwise estimate holds:

$$
u(x, t) \geq \frac{\underline{\kappa}}{(t+\underline{\tau})^{\frac{1}{m-1}}}\left(\frac{1}{(r+\underline{R})^{\nu-1}}-\frac{\underline{\gamma}}{[\log (t+\underline{\tau})]^{\frac{\nu-1}{\nu+1}}}\right)_{+}^{\frac{1}{m-1}} \quad \forall x \equiv(r, \theta) \in \mathbb{M}^{n} \backslash B_{1}, \forall t \geq 0,
$$

where $\underline{\kappa}, \underline{\gamma}, \underline{R}, \underline{\tau}$ are positive constants depending only on $n, m, \nu, c, R, u_{0}$ and on the metric of $\mathbb{M}^{n}$. In particular there hold

$$
\|u(\cdot, t)\|_{\infty} \geq \frac{\underline{K}}{t^{\frac{1}{m-1}}} \quad \text { and } \quad \underline{\mathrm{R}}(t) \geq \underline{\Gamma}(\log t)^{\frac{1}{1+\nu}} \quad \forall t \geq \underline{T},
$$

where $\underline{\mathrm{R}}(t)$ is the radius of the largest ball contained in the support of $u(\cdot, t)$ and $\underline{K}, \underline{\Gamma}, \underline{T}$ are positive constants as above.

The proofs of Theorems 3.7 and 3.8 follow by barrier arguments very similar to those exploited in the proofs of Theorems 3.3 and 3.4, for whose details we refer to [13]. Indeed, the algebra of computations is essentially the same, since the factors inside positive parts in (3.37) and (3.39) do coincide with those in (3.5) and (3.7), respectively, except for a sign change in order to preserve monotonicity with respect to $r$ and $t$. Another (inessential) difference is due to the fact that in (3.37) and (3.39) the parameters $\bar{R}$ and $\underline{R}$ appear in the barrier itself; this is basically a readability choice, since global (in space) barriers in (3.5) and (3.7) could also be written, but their explicit form would be more complicated (recall (3.9)). On top of that, our main interest is in describing the behavior of solutions where $r$ and $t$ are large.

As concerns the upper bound on the $L^{\infty}\left(\mathbb{M}^{n}\right)$ norm provided by (3.38), in Section 4 we will see that it can actually be improved to become an absolute bound, namely the same estimate with a constant $\bar{K}$ which is independent of the initial datum.

3.3. Critical curvatures and the quasi-Euclidean range. According to the ranges established in Subsection 2.3, we still have to consider critical curvatures, that is those corresponding to the boundaries between the quasi-hyperbolic range and the super-hyperbolic and quasi-Euclidean ranges.

Theorem 3.9 (Upper bounds, quasi-hyperbolic critical, Theorem 3.3 of [12]). Assume that (3.1) is satisfied for some $C, R>0$ and $\mu=1$. Let $u$ be the solution of (1.1) starting from a nonnegative, bounded and compactly-supported initial datum. Then the following pointwise estimate holds:

$$
u(x, t) \leq \frac{\bar{\kappa}}{(t+\bar{\tau})^{\frac{1}{m-1}}}\left[\bar{\eta}+\frac{1}{2} \log \log (t+\bar{\tau})-\log r\right]_{+}^{\frac{1}{m-1}} \quad \forall x \equiv(r, \theta) \in \mathbb{M}^{n} \backslash B_{\bar{R}}, \forall t \geq 0,
$$

where $\bar{\kappa}, \bar{\eta}, \bar{R}, \bar{\tau}$ are positive constants depending only on $n, m, C, R, u_{0}$. In particular there hold

$$
\|u(\cdot, t)\|_{\infty} \leq \bar{K}\left(\frac{\log \log t}{t}\right)^{\frac{1}{m-1}} \quad \text { and } \quad \overline{\mathrm{R}}(t) \leq \bar{\Gamma}(\log t)^{\frac{1}{2}} \quad \forall t \geq \bar{T},
$$

where $\overline{\mathrm{R}}(t)$ is the radius of the smallest ball containing the support of $u(\cdot, t)$ and $\bar{K}, \bar{\Gamma}, \bar{T}$ are positive constants as above.

Theorem 3.10 (Lower bounds, quasi-hyperbolic critical, Theorem 3.4 of [12]). Assume that (3.2) is satisfied for some $c, R>0$ and $\nu=1$. Let $u$ be the solution of (1.1) starting from a nonnegative, bounded, compactly-supported and nontrivial initial datum. Then the following pointwise estimate holds:

$$
u(x, t) \geq \frac{\underline{\kappa}}{(t+\underline{\tau})^{\frac{1}{m-1}}}\left[-\underline{\eta}+\frac{1}{2} \log \log (t+\underline{\tau})-\log r\right]_{+}^{\frac{1}{m-1}} \quad \forall x \equiv(r, \theta) \in \mathbb{M}^{n} \backslash B_{\underline{R}}, \forall t \geq 0,
$$

where $\underline{\kappa}, \underline{\eta}, \underline{R}, \underline{\tau}$ are positive constants depending only on $n, m, c, R, u_{0}$ and on the metric of $\mathbb{M}^{n}$. In particular there hold

$$
\|u(\cdot, t)\|_{\infty} \geq \underline{K}\left(\frac{\log \log t}{t}\right)^{\frac{1}{m-1}} \quad \text { and } \quad \underline{\mathrm{R}}(t) \geq \underline{\Gamma}(\log t)^{\frac{1}{2}} \quad \forall t \geq \underline{T},
$$

where $\underline{\mathrm{R}}(t)$ is the radius of the largest ball contained in the support of $u(\cdot, t)$ and $\underline{K}, \underline{\Gamma}, \underline{T}$ are positive constants as above.

Specific comments regarding Theorems 3.9 and 3.10, along with all the other results stated below, will be made at the end of this subsection. We now turn to the critical case corresponding to the boundary between the quasi-hyperbolic and the quasi-Euclidean region. 
Theorem 3.11 (Upper bounds, quasi-Euclidean critical, Theorem 6.3 of [12]). Assume that (3.1) is satisfied for some $C, R>0$ and $\mu=-1$. Let $u$ be the solution of (1.1) starting from a nonnegative, bounded and compactly-supported initial datum. Let

$$
q:=\frac{1+\sqrt{1+4 C}}{2}, \quad n_{q}:=1+q(n-1) .
$$

Then the following pointwise estimate holds:

$$
u(x, t) \leq \frac{\bar{\kappa}}{(t+\bar{\tau})^{\frac{n_{q}}{2+n_{q}(m-1)}}}\left[\bar{\gamma}-\frac{r^{2}}{(t+\bar{\tau})^{\frac{2}{2+n_{q}(m-1)}}}\right]_{+}^{\frac{1}{m-1}} \quad \forall x \equiv(r, \theta) \in \mathbb{M}^{n}, \forall t \geq 0,
$$

where $\bar{\kappa}, \bar{\gamma}, \bar{\tau}$ are positive constants depending only on $n, m, C, R, u_{0}$. In particular there hold

$$
\|u(\cdot, t)\|_{\infty} \leq \frac{\bar{K}}{t^{\frac{n_{q}}{2+n_{q}(m-1)}}} \quad \text { and } \quad \overline{\mathrm{R}}(t) \leq \frac{\bar{\Gamma}}{t^{\frac{1}{2+n_{q}(m-1)}}} \quad \forall t \geq \bar{T},
$$

where $\overline{\mathrm{R}}(t)$ is the radius of the smallest ball containing the support of $u(\cdot, t)$ and $\bar{K}, \bar{\Gamma}, \bar{T}$ are positive constants as above.

Theorem 3.12 (Lower bounds, quasi-Euclidean critical, Theorem 6.4 of [12]). Assume that (3.2) is satisfied for some $c, R>0$ and $\nu=-1$. Let $u$ be the solution of (1.1) starting from a nonnegative, bounded, compactly-supported and nontrivial initial datum. Then the following pointwise estimate holds:

$$
u(x, t) \geq \frac{\underline{\kappa}}{(t+\underline{\tau})^{\frac{n_{q}}{2+n_{q}(m-1)}}}\left[\underline{\gamma}-\frac{r^{2}}{(t+\underline{\tau})^{\frac{2}{2+n_{q}(m-1)}}}\right]_{+}^{\frac{1}{m-1}} \quad \forall x \equiv(r, \theta) \in \mathbb{M}^{n}, \forall t \geq 0,
$$

where $q$ and $n_{q}$ are as in (3.41), with $C$ replaced by $c$, while $\underline{\kappa}, \underline{\gamma}, \underline{\tau}$ are positive constants depending only on $n, m, c, R, u_{0}$ and on the metric of $\mathbb{M}^{n}$. In particular there hold

$$
\|u(\cdot, t)\|_{\infty} \geq \frac{\frac{K}{n_{q}}}{t^{\frac{2+n_{q}(m-1)}{2}}} \quad \text { and } \quad \underline{\mathrm{R}}(t) \geq \frac{\underline{\Gamma}}{t^{\frac{1}{2+n_{q}(m-1)}}} \quad \forall t \geq \underline{T},
$$

where $\underline{\mathrm{R}}(t)$ is the radius of the largest ball contained in the support of $u(\cdot, t)$ and $\underline{K}, \underline{\Gamma}, \underline{T}$ are positive constants as above.

We conclude by addressing the quasi-Euclidean range, where the curvature decays fast at infinity, that is like a negative power of the distance which is more than quadratic.

Theorem 3.13 (Upper bounds, quasi-Euclidean, Theorem 6.1 of [12]). Assume that (3.1) is satisfied for some $C, R>0$ and $\mu<-1$. Let $u$ be the solution of (1.1) starting from a nonnegative, bounded and compactly-supported initial datum. Then the following pointwise estimate holds:

$$
u(x, t) \leq \frac{\bar{\kappa}}{(t+\bar{\tau})^{\frac{n}{2+n(m-1)}}}\left[\bar{\gamma}-\frac{r^{2}}{(t+\bar{\tau})^{\frac{2}{2+n(m-1)}}}\right]_{+}^{\frac{1}{m-1}} \quad \forall x \equiv(r, \theta) \in \mathbb{M}^{n}, \forall t \geq 0,
$$

where $\bar{\kappa}, \bar{\gamma}, \bar{\tau}$ are positive constants depending only on $n, m, \mu, C, R, u_{0}$. In particular there hold

$$
\|u(\cdot, t)\|_{\infty} \leq \frac{\bar{K}}{t^{\overline{2+n(m-1)}}} \quad \text { and } \quad \overline{\mathrm{R}}(t) \leq \frac{\bar{\Gamma}}{t^{\frac{1}{2+n(m-1)}}} \quad \forall t \geq \bar{T},
$$

where $\overline{\mathrm{R}}(t)$ is the radius of the smallest ball containing the support of $u(\cdot, t)$ and $\bar{K}, \bar{\Gamma}, \bar{T}$ are positive constants as above.

Theorem 3.14 (Lower bounds, quasi-Euclidean, Theorem 6.2 of [12]). Assume that (3.2) is satisfied for some $c, R>0$ and $\nu<-1$. Let $u$ be the solution of (1.1) starting from a nonnegative, bounded, compactly-supported and nontrivial initial datum. Then the following pointwise estimate holds:

$$
u(x, t) \geq \frac{\underline{\kappa}}{(t+\underline{\tau})^{\frac{n}{2+n(m-1)}}}\left[\underline{\gamma}-\frac{r^{2}}{(t+\underline{\tau})^{\frac{2}{2+n(m-1)}}}\right]_{+}^{\frac{1}{m-1}} \quad \forall x \equiv(r, \theta) \in \mathbb{M}^{n}, \forall t \geq 0,
$$

where $\underline{\kappa}, \underline{\gamma}, \underline{\tau}$ are positive constants depending only on $n, m, \nu, c, R, u_{0}$ and on the metric of $\mathbb{M}^{n}$. In particular there hold

$$
\|u(\cdot, t)\|_{\infty} \geq \frac{\underline{K}}{t^{\frac{n}{2+n(m-1)}}} \quad \text { and } \quad \underline{\mathrm{R}}(t) \geq \frac{\underline{\Gamma}}{t^{\frac{1}{2+n(m-1)}}} \quad \forall t \geq \underline{T},
$$

where $\underline{\mathrm{R}}(t)$ is the radius of the largest ball contained in the support of $u(\cdot, t)$ and $\underline{K}, \underline{\Gamma}, \underline{T}$ are positive constants as above. 
A few remarks regarding the above results are in order.

- The proof of Theorem 3.9 carried out in [12] is obtained by keeping track of the dependence of $\bar{\kappa}$ and $\bar{\gamma}$ on the power $\mu$ and then letting $\mu \uparrow 1$ in (3.5). This causes the power $1-\mu$ to become a logarithm, whence estimate (3.40). The same holds for Theorem 3.10, which in particular ensures the sharpness of the bounds also in the (delicate) quasi-hyperbolic critical case.

- In the quasi-Euclidean critical case, Theorems 3.11 and 3.12 show a Euclidean-like behavior up to changing the actual dimension, that switches from $n$ to $n_{q}>n$. Note that these are the sole results above where the value of the constants $C$ and $c$ appearing in the curvature bounds (3.1) and (3.2), respectively, plays a significant role, affecting qualitatively the solution profiles. As regards the corresponding proofs provided in [12], we mention that they are performed by means of a suitable radial change of variables, which turns diffusion on a model manifold $\mathbb{M}^{n}$ into diffusion on $\mathbb{R}^{n}$ with a weight exhibiting a power-type behavior at infinity. Such a change of variables was first introduced in [25, Section 6] and then generalized in [12, Section 7]. One can thus reduce (1.1) to a weighted Euclidean problem that was studied previously: see [21, 15, 20].

- As the reader might notice, Theorems 3.13 and 3.14 entail that there is no qualitative difference between the behavior of solutions on quasi-Euclidean manifolds and on $\mathbb{R}^{n}$. In other words, as soon as the curvature vanishes sufficiently fast at infinity (like a more-than-quadratic negative power of the distance), we can claim that diffusion is barely affected by the geometry of $\mathbb{M}^{n}$, at least at the level of time decay and support properties of the solutions (i.e. speed of propagation).

- It is remarkable that, though the approximate shapes of the solutions vary, the expansion rate of the support always obeys to the asymptotic law $\asymp(\log t)^{1 /(1+\mu)}$, at least for $\mu>-1$, namely all the way from the quasi-hyperbolic to the super-hyperbolic range. On the other hand, the behavior of the $L^{\infty}\left(\mathbb{M}^{n}\right)$ norm exhibits more significant changes passing from one region to the other. We will try to give a partial (functional) explanation of such a phenomenon in Section 5 .

\section{Asymptotics in the CASE OF VERY NEgAtive CURVATURE}

The aim of this section is to describe, in a more precise way, the asymptotic behavior of the solutions to (1.1) as $t \rightarrow \infty$ in the super-hyperbolic range. Theorems 3.7 and 3.8 provide important information on the decay rate of the $L^{\infty}\left(\mathbb{M}^{n}\right)$ norm and on the expansion rate of the support; however, they lack a convergence result towards an appropriate profile, which is still proved in [13].

4.1. A sublinear elliptic equation: existence and qualitative properties. In order to investigate the convergence properties of (bounded) solutions to (1.1) in the super-hyperbolic range, first of all we need to analyze the following sublinear elliptic equation:

$$
-\Delta\left(v^{m}\right)=\frac{1}{m-1} v \quad \text { on } \mathbb{M}^{n},
$$

where $v$ is meant to be a nonnegative, bounded and nontrivial function. The adjective "sublinear" comes from the fact that, upon the change of variables $v=v^{m}$, the zero-order term in (4.1) becomes a power of $v$ which is strictly less than 1 (recall that $m>1$ ). If a solution to (4.1) exists, an elementary computation shows that it gives rise to a separable solution of the differential equation in (1.1) of the form

$$
(x, t) \mapsto t^{-\frac{1}{m-1}} v(x) .
$$

Such a solution, as we will see in Subsection 4.2, will play the role of an attractor for general solutions to (1.1), thus reproducing the same type of asymptotics that occurs on bounded Euclidean domains [22].

In agreement with the simplified approach to the notion of solution, that we discussed in the beginning of Section 2 with regards to (1.1), in the sequel by "solution" to (4.1) we will mean the one constructed through the usual approximation by homogeneous Dirichlet problems on balls, that is (let $k \in \mathbb{N}$ )

$$
\begin{cases}-\Delta\left(v_{k}^{m}\right)=\frac{1}{m-1} v_{k} & \text { in } B_{k} \\ v_{k}=0 & \text { on } \partial B_{k} .\end{cases}
$$

If a solution exists as $k \rightarrow \infty$, then it is usually referred to as minimal, since it actually turns out to be smaller than any other positive solution. Nevertheless, a priori it is not for granted that $\left\{v_{k}\right\}$ converges at all to a solution of (4.1). In fact, this is part of the proof of the next result.

Theorem 4.1 (Sublinear elliptic equation, Theorem 2.2 of [13]). Assume that (3.1) is satisfied for some $C, R>0$ and $\mu>1$. Then there exists a solution $v$ of (4.1) which is positive, bounded, smooth and complies with the upper estimate

$$
\limsup _{r \rightarrow \infty} r^{\frac{\mu-1}{m-1}} v(x) \leq[m(\mu-1)(n-1) \sqrt{C}]^{-\frac{1}{m-1}}
$$


If in addition (3.2) is satisfied for some $c>0$ and $\nu \geq \mu$, then $v$ also complies with the lower estimate

$$
\liminf _{r \rightarrow \infty} r^{\frac{\nu-1}{m-1}} v(x) \geq[m(\nu-1)(n-1) \sqrt{c}]^{-\frac{1}{m-1}} .
$$

The real statement of Theorem 4.1 in [13] is more complicated (see Remark 4.5 below for some details). Nevertheless, the above simplified statement is enough for the purposes of the present paper.

It is worth pointing out that (4.4) provides a sharp decay rate for $v$ at infinity, even with respect to the multiplying constant. Indeed, thanks to (4.5) we deduce that the rate is achieved on model manifolds attaining equality outside a ball in the curvature bounds (3.1) and (3.2) (recall Subsection 2.1).

The proof of Theorem 4.1 we will provide is still based on delicate barriers. To this aim, we need two preliminary results, whose proofs (that we omit) follow arguments similar to those used in Section 3.

Lemma 4.2 (Lemma 3.3 of [13]). Assume that (3.1) is satisfied for some $C, R>0$ and $\mu>1$. Then there exist positive constants $\bar{R}, \bar{\kappa}_{0}$, depending only on $n, m, \mu, C, R$, such that the function

$$
\bar{v}(x) \equiv \bar{v}(r):=\frac{\bar{\kappa}}{\left(r^{2}+\bar{R}^{2}\right)^{\frac{\mu-1}{2(m-1)}}} \quad \forall x \equiv(r, \theta) \in \mathbb{M}^{n}
$$

is a supersolution to (4.1) for all $\bar{\kappa} \geq \bar{\kappa}_{0}$, i.e. it complies with

$$
-\Delta\left(\bar{v}^{m}\right) \geq \frac{1}{m-1} \bar{v} \quad \text { on } \mathbb{M}^{n} .
$$

Lemma 4.3 (Lemma 3.4 of [13]). Assume that (3.2) is satisfied for some $c, R>0$ and $\nu>1$. Let $\varepsilon, \delta>0$. Then there exists a positive constant $\underline{R}$, depending only on $n, m, \nu, c, R, \varepsilon$ and on the infimum of the Ricci curvature of $\mathbb{M}^{n}$ in $B_{R}$, such that the function

$\underline{v}(x) \equiv \underline{v}(r):=\underline{\kappa}_{\varepsilon}\left[\frac{1}{\left(r^{2}+\underline{R}^{2}\right)^{\frac{\nu-1}{2}}}-\delta\right]_{+}^{\frac{1}{m-1}} \quad \forall x \equiv(r, \theta) \in \mathbb{M}^{n}, \quad \underline{\kappa}_{\varepsilon}:=[m(\nu-1)(n-1) \sqrt{c+\varepsilon}]^{-\frac{1}{m-1}}$,

is a subsolution to (4.1), i.e. it complies with

$$
-\Delta\left(\underline{v}^{m}\right) \leq \frac{1}{m-1} \underline{v} \quad \text { on } \mathbb{M}^{n} .
$$

Proof of Theorem 4.1. Let $\left\{v_{k}\right\}$ be the solutions to (4.3). The latter do exist and have good properties such as inner regularity, boundedness and strict positivity inside each $B_{k}$; moreover, since $v_{k+1}$ solves the same elliptic equation as $v_{k}$ in $B_{k}$, but on $\bar{B}_{k}$ is strictly positive, the (local) comparison principle ensures that $v_{k} \leq v_{k+1}$ in $B_{k}$. These are standard results in $\mathbb{R}^{n}$, for which the best reference is [4]. The fact that here we work on Riemannian manifolds is inessential, such properties being purely local.

If (3.1) holds, then Lemma 4.2 and the just mentioned comparison principle ensure that

$$
v_{k}(x) \leq \frac{\bar{\kappa}}{\left(r^{2}+\bar{R}^{2}\right)^{\frac{\mu-1}{2(m-1)}}} \quad \forall x \equiv(r, \theta) \in B_{k} .
$$

Estimate (4.7) is crucial, because it entails the uniform boundedness of the sequence $\left\{v_{k}\right\}$. This is the key point where (3.1) comes into play, as in general we cannot guarantee that the sequence stays bounded. Consequently, recalling that $\left\{v_{k}\right\}$ is monotone increasing (each $v_{k}$ is implicitly extended to zero outside $B_{k}$ ), we can claim that it admits a pointwise limit $v$ which also satisfies

$$
v(x):=\lim _{k \rightarrow \infty} v_{k}(x) \leq \frac{\bar{\kappa}}{\left(r^{2}+\bar{R}^{2}\right)^{\frac{\mu-1}{2(m-1)}}} \quad \forall x \equiv(r, \theta) \in \mathbb{M}^{n} .
$$

The fact that $v$ is indeed a solution to (4.1) follows directly from the construction procedure: each $v_{k}$ solves the equation in $B_{k}$ and the sequence converges to $v$ as $k \rightarrow \infty$, hence one can pass to the limit in the so-called very weak formulation (i.e. the entire Laplacian is moved to the test function). Alternatively, the uniform boundedness and boundedness away from zero of $\left\{v_{k}\right\}$ plus elliptic regularity estimates ensure, for instance, that the sequence is also locally bounded in $C^{2, \alpha}\left(\mathbb{M}^{n}\right)$, which is enough to pass to the limit in the classical formulation of (4.1) by Ascoli-Arzelà. For more details, see again [4].

We now turn to the proof of (4.4). To this aim, let us consider the model function $\psi$ provided by Lemma 3.1 and denote by $V(r)$ the positive, bounded, smooth solution to

$$
\left(\psi^{n-1}\left(V^{m}\right)^{\prime}\right)^{\prime}=-\frac{1}{m-1} \psi^{n-1} V \quad \text { in }(0, \infty)
$$

which vanishes as $r \rightarrow \infty$, where for simplicity ' stands for derivative with respect to $r$. Note that such a solution does exist in view of what we have proved above: it is nothing but the solution of (4.1) on 
the model manifold associated to $\psi$ (recall identity $(2.3)$ ), which is radial due to the radially-symmetric structure of model manifolds. Upon integrating (4.9) from 0 to $\varrho>0$, we obtain:

$$
\left(V^{m}\right)^{\prime}(\varrho)=-\frac{1}{(m-1) \psi(\varrho)^{n-1}} \int_{0}^{\varrho} \psi(s)^{n-1} V(s) d s \quad \forall \varrho>0 .
$$

A further integration of (4.10) from $r$ to $\infty$ yields

$$
V(r)^{m}=\frac{1}{m-1} \int_{r}^{\infty}\left(\frac{1}{\psi(\varrho)^{n-1}} \int_{0}^{\varrho} \psi(s)^{n-1} V(s) d s\right) d \varrho \quad \forall r>0,
$$

where we have exploited the fact that $V$ vanishes at infinity. As a starting step of an iterative procedure, we assume that the bound

$$
\limsup _{r \rightarrow \infty} r^{\frac{\mu-1}{m-1}} V(r) \leq \kappa
$$

holds for some $\kappa>0$. We know, from (4.8) applied to $v \equiv V$, that (4.12) is indeed satisfied with $\kappa=\bar{\kappa}$. Thanks to (3.4), (4.12) and integration by parts, for every $0<\varepsilon<C$ there exists $\varrho_{\varepsilon}>0$ such that:

$$
\begin{aligned}
\int_{\varrho_{\varepsilon}}^{\varrho} \psi(s)^{n-1} V(s) d s & \stackrel{(4.12)}{\leq}(\kappa+\varepsilon) \int_{\varrho_{\varepsilon}}^{\varrho} \psi(s)^{n-1} s^{-\frac{\mu-1}{m-1}} d s \\
& \stackrel{(3.4)}{\leq} \frac{\kappa+\varepsilon}{\sqrt{C-\varepsilon}} \int_{\varrho_{\varepsilon}}^{\varrho} \psi(s)^{n-2} \psi^{\prime}(s) s^{-\frac{\mu m-1}{m-1}} d s \\
& \text { i.b.p. } \frac{\kappa+\varepsilon}{(n-1) \sqrt{C-\varepsilon}}\left[\psi(\varrho)^{n-1} \varrho^{-\frac{\mu m-1}{m-1}}+\frac{\mu m-1}{m-1} \int_{\varrho_{\varepsilon}}^{\varrho} \psi(s)^{n-1} s^{-\frac{(\mu+1) m-2}{m-1}} d s\right] \\
& m>1 \\
& \leq \frac{\kappa+\varepsilon}{(n-1) \sqrt{C-\varepsilon}} \psi(\varrho)^{n-1} \varrho^{-\frac{\mu m-1}{m-1}}+\varepsilon \int_{\varrho_{\varepsilon}}^{\varrho} \psi(s)^{n-1} s^{-\frac{\mu-1}{m-1}} d s \quad \forall \varrho>\varrho_{\varepsilon} .
\end{aligned}
$$

From (4.13) we can therefore deduce that

$$
\int_{\varrho_{\varepsilon}}^{\varrho} \psi(s)^{n-1} V(s) d s \leq \frac{(\kappa+\varepsilon)^{2}}{\kappa(n-1) \sqrt{C-\varepsilon}} \psi(\varrho)^{n-1} \varrho^{-\frac{\mu m-1}{m-1}} \quad \forall \varrho>\varrho_{\varepsilon} .
$$

By plugging estimate (4.14) in (4.11) we obtain, for all $r>\varrho_{\varepsilon}$, the bound

$$
V(r)^{m} \leq \frac{1}{m-1} \int_{r}^{\infty}\left(\frac{1}{\psi(\varrho)^{n-1}} \int_{0}^{\varrho_{\varepsilon}} \psi(s)^{n-1} V(s) d s\right) d \varrho+\frac{(\kappa+\varepsilon)^{2}}{\kappa m(\mu-1)(n-1) \sqrt{C-\varepsilon}} r^{-m \frac{\mu-1}{m-1}},
$$

whence, upon multiplying by $r^{\frac{m-1}{m-1}}$, letting first $r \rightarrow \infty$ and then $\varepsilon \rightarrow 0$, we end up with

$$
\limsup _{r \rightarrow \infty} r^{m \frac{\mu-1}{m-1}} V(r)^{m} \leq \frac{\kappa}{m(\mu-1)(n-1) \sqrt{C}}
$$

Note that we have implicitly exploited the fact that the integral in (4.15) vanishes faster than an exponential as $r \rightarrow \infty$, due to (3.4). As a consequence of (4.16), we therefore infer that (4.12) also holds with $\kappa$ replaced by the new constant

$$
\left[\frac{\kappa}{m(\mu-1)(n-1) \sqrt{C}}\right]^{\frac{1}{m}} .
$$

This shows how the iterative procedure goes on, and it is not difficult to check that after an infinite number of steps we obtain the bound

$$
\limsup _{r \rightarrow \infty} r^{\frac{\mu-1}{m-1}} V(r) \leq[m(\mu-1)(n-1) \sqrt{C}]^{-\frac{1}{m-1}}
$$

In order to prove (4.4) it is enough to observe that, $V(r) \equiv V(x)$ being a positive, radially-decreasing function (recall (4.10)) that complies with (4.9), by Laplacian comparison it turns out to be a supersolution to (4.1), namely (4.6) holds with $\bar{v}$ replaced by $V$. Arguing as in the first part of the proof, we thus deduce that $v \leq V$, whence (4.4) in view of (4.17).

Finally, suppose that also the lower curvature bound (3.2) is satisfied (the request $\nu \geq \mu$ is made just for compatibility). Thanks to Lemma 4.3 and once again the comparison principle applied in $B_{k}$, we can assert that for every $\varepsilon, \delta>0$ there holds

$$
v_{k}(x) \geq[m(\nu-1)(n-1) \sqrt{c+\varepsilon}]^{-\frac{1}{m-1}}\left[\frac{1}{\left(r^{2}+\underline{R}^{2}\right)^{\frac{\nu-1}{2}}}-\delta\right]_{+}^{\frac{1}{m-1}} \quad \forall x \equiv(r, \theta) \in B_{k},
$$


provided $k$ is so large that $\operatorname{supp} \underline{v}$ is entirely contained in $B_{k}$. Estimate (4.5) follows from (4.18) upon letting first $k \rightarrow \infty$, then $\delta \rightarrow 0$, multiplying by $r^{(\nu-1) /(m-1)}$, letting $r \rightarrow \infty$ and finally $\varepsilon \rightarrow 0$.

4.2. Asymptotics for bounded solutions to the PME. As we disclosed in the beginning of this section, the solution of the sublinear elliptic equation (4.1) that we constructed above has a key role in the description of the asymptotic behavior of solutions to (1.1). This is the content of the next result.

Theorem 4.4 (Asymptotic convergence, super-hyperbolic, Theorem 2.4 of [13]). Assume that (3.1) is satisfied for some $C, R>0$ and $\mu>1$. Let $u$ be the solution of (1.1) starting from a nonnegative, bounded and nontrivial initial datum. Let $v$ be the solution of (4.1) provided by Theorem 4.1. Then

$$
\lim _{t \rightarrow \infty}\left\|t^{\frac{1}{m-1}} u(\cdot, t)-v\right\|_{\infty}=0
$$

Moreover, $u$ complies with the following upper bound:

$$
u(x, t) \leq \frac{v(x)}{t^{\frac{1}{m-1}}} \quad \forall(x, t) \in \mathbb{M}^{n} \times(0, \infty) ;
$$

in particular, there holds the absolute bound

$$
\|u(\cdot, t)\|_{\infty} \leq \frac{\|v\|_{\infty}}{t^{\frac{1}{m-1}}} \quad \forall t>0
$$

In the Euclidean framework, several analogues of Theorem 4.4 are available: see [22, Theorem 1.1] or [24, Theorem 20.1] in the case of bounded domains (with homogeneous Dirichlet boundary conditions), [15, Theorem 5.1] in the case of a weighted PME and [10, Theorem 3.1] for its fractional counterpart. In the whole of $\mathbb{R}^{n}$, without a weight that decays sufficiently fast, the result is false since asymptotics is governed by Barenblatt-type profiles (see the Introduction). The strategy of proof is by now classical and it mostly relies on the arguments developed in [22], so we will be fairly concise.

Proof of Theorem 4.4. We know that the separable solution (4.2) is indeed a solution to the differential equation in (1.1), whose initial condition at $t=0$ is everywhere $\infty$ since $v$ is strictly positive. By the comparison principle, which should be rigorously applied in $B_{k} \times(0, \infty)$ to the solutions of (2.1) upon a passage to the limit as $k \rightarrow \infty$, we therefore deduce the validity of (4.20). The absolute bound (4.21) is then a consequence of the global boundedness of $v$. In order to prove (4.19), it is convenient to introduce the following change of variables:

$$
u(x, t)=e^{-\frac{\tau}{m-1}} U(x, \tau) \quad \text { with } \quad t=e^{\tau} \quad \forall(x, t, \tau) \in \mathbb{M}^{n} \times(0, \infty) \times(-\infty, \infty) .
$$

It is easily checked that $U$ is a (nonnegative) solution to the equation

$$
U_{\tau}=\Delta\left(U^{m}\right)+\frac{1}{m-1} U \quad \text { on } \mathbb{M}^{n} \times(-\infty, \infty) ;
$$

moreover, in terms of $U$, estimates (4.20) and (4.21) read

$$
U(x, \tau) \leq v(x) \leq\|v\|_{\infty} \quad \forall(x, \tau) \in \mathbb{M}^{n} \times(-\infty, \infty) .
$$

Now we need to invoke a major result for positive solutions to (1.1), due to Ph. Bénilan and M.G. Crandall. They proved in [1] that such solutions satisfy the following inequality:

$$
u_{t} \geq-\frac{u}{(m-1) t} \quad \text { on } \mathbb{M}^{n} \times(0, \infty) \text {. }
$$

In fact the framework of [1] is Euclidean, but actually (4.25) only depends on time-scaling properties of the PME (see e.g. [24, Page 182]), hence it can plainly be extended to the manifold setting. Note that (4.25) is equivalent to

$$
U_{\tau} \geq 0 \quad \text { on } \mathbb{M}^{n} \times(-\infty, \infty),
$$

so that the map $\tau \mapsto U(x, \tau)$ is monotone non-decreasing for all $x \in \mathbb{M}^{n}$. By virtue of (4.26) and the bound (4.24), we infer that there exists the pointwise limit $\hat{v}(x):=\lim _{\tau \rightarrow \infty} U(x, \tau)$, which satisfies

$$
0 \leq \hat{v} \leq v, \quad \hat{v} \not \equiv 0 .
$$

We point out that $\hat{v}$ is nontrivial because so is the initial datum $u_{0}$. We are left with establishing that $\hat{v}$ is indeed a solution to (4.1). To this purpose, let us integrate (4.23) between $\tau$ and $\tau+1$ :

$$
U(x, \tau+1)-U(x, \tau)=\Delta \int_{\tau}^{\tau+1} U(x, s)^{m} d s+\frac{1}{m-1} \int_{\tau}^{\tau+1} U(x, s) d s ;
$$

it is apparent that, by the above monotonicity property, there hold

$$
U(x, \tau+1)-U(x, \tau) \stackrel{\tau \rightarrow \infty}{\longrightarrow} 0, \quad \int_{\tau}^{\tau+1} U(x, s)^{m} d s \stackrel{\tau \rightarrow \infty}{\longrightarrow} \hat{v}(x)^{m}, \quad \int_{\tau}^{\tau+1} U(x, s) d s \stackrel{\tau \rightarrow \infty}{\longrightarrow} \hat{v}(x) .
$$


In view of (4.29), we can therefore pass to the limit in (4.28) and deduce that $\hat{v}$ does satisfy (4.1), thus it is strictly positive by local elliptic regularity. In fact the Laplacian term should be handled more carefully, by using the very weak formulation of (4.23) (for the details we refer to the original proof in [13]). Thanks to (4.27), we also know that $\hat{v} \leq v$ : however, since $v$ turns out to be minimal, i.e. it is the smallest positive solution of (4.1), this implies $\hat{v} \equiv v$.

We have therefore proved that $U(\cdot, \tau)$ converges pointwise to $v$ as $\tau \rightarrow \infty$. The fact that convergence actually occurs locally uniformly is a consequence of local Hölder-regularity properties of bounded solutions to the PME combined with Ascoli-Arzelà: see again [13] and references therein for more details. On the other hand, estimate (4.24) plus (4.4) yield a uniform decay rate of $U(\cdot, \tau)$ at spatial infinity, which allows one to pass from local to global uniform convergence. By undoing the change of variables (4.22), it is readily seen that this result in terms of $u$ is exactly (4.19).

Remark 4.5 (Uniqueness, non-uniqueness and comparison issues). In fact the theses of Theorems 2.2 and 2.4 of [13] are more general than those we stated here. Indeed, it is shown that the solution of (4.1) we constructed in Theorem 4.1 is actually unique in the class of solutions vanishing at infinity, i.e. satisfying $\lim _{r \rightarrow \infty} v(x)=0$, in addition to being the minimal one. This is the consequence of a comparison principle at infinity, which asserts that if a positive supersolution $\bar{v}$ and a nonnegative subsolution $\underline{v}$ are "ordered at infinity" then they are ordered everywhere. On the other hand, it is also proved that if this assumption is dropped uniqueness fails, since one can construct multiple solutions to (4.1) that do not vanish at infinity. Finally, we point out that similar uniqueness and comparison results hold for the solutions to (1.1), see Theorem 2.5 of [13].

\section{New Sobolev-type inequalities And Smoothing efFects}

The sharp pointwise bounds that we obtained in Section 3, through pure barrier methods, also have a clear functional-analytic interpretation which we describe below, at least at the level of asymptotic behavior of the $L^{\infty}\left(\mathbb{M}^{n}\right)$ norm and in the "left" range of curvature (i.e. when $\mu \leq 0$ ). This is strictly related to the validity of suitable Sobolev-type inequalities, as firstly observed in [8].

5.1. A new family of radial Sobolev-type inequalities. The main result of [19], which we now state, establishes that if the curvature of $\mathbb{M}^{n}$ decays at infinity like a negative power of the distance that is less than quadratic, then there holds a suitable family of Sobolev-type inequalities for radial functions, including all the exponents from $2^{*}$ to 2 except the latter.

Theorem 5.1 (Sobolev-type inequalities, left quasi-hyperbolic, Theorem 2.1 of [19]). Assume that (3.1) is satisfied for some $C, R>0$ and $\mu \in(-1,0)$. Then there exists a positive constant $\mathrm{C}$, depending only on $n, \mu, C, R$, such that there hold the radial Sobolev-type inequalities

$$
\|f\|_{L^{p}\left(\mathbb{M}^{n}\right)} \leq \mathrm{C} p^{\frac{1-\mu}{2(1+\mu)}}(p-2)^{\frac{\mu}{1+\mu}}\|\nabla f\|_{L^{2}\left(\mathbb{M}^{n}\right)} \quad \forall f \in C_{c: \operatorname{rad}}^{1}\left(\mathbb{M}^{n}\right), \quad \forall p \in\left(2,2^{*}\right] \cap(2, \infty) .
$$

The proof of Theorem 5.1 given in [19] relies on Laplacian comparison and on the possibility to write (5.1) as a weighted Euclidean inequality. The result is sharp, since on model manifolds attaining equality in (3.1) the best Sobolev constant does behave like the right-hand side of (5.1) as $p \rightarrow 2$ (see again [19]).

In the hyperbolic case $\mu=0$ also the Poincaré inequality holds $(p=2)$, and even for nonradial functions. This is proved in [19, Theorem 5.5], which is in fact a minor extension of McKean's theorem, that we briefly discussed in Subsection 2.2.

If the curvature decay of $\mathbb{M}^{n}$ is quadratic, i.e. we are in the quasi-Euclidean critical case $(\mu=-1)$, we still have radial Sobolev-type inequalities. However, the lower exponent is no more 2 but depends in a nontrivial way on the multiplicative constant $C$ (in agreement with Theorems 3.11 and 3.12).

Theorem 5.2 (Sobolev-type inequalities, quasi-Euclidean critical, Theorem 2.2 of [19]). Assume that (3.1) is satisfied for some $C, R>0$ and $\mu=-1$. Then there exists a positive constant $\mathrm{C}$, depending only on $n, C, R$, such that there hold the radial Sobolev-type inequalities

$$
\|f\|_{L^{p}\left(\mathbb{M}^{n}\right)} \leq \mathrm{C} \sqrt{p}\|\nabla f\|_{L^{2}\left(\mathbb{M}^{n}\right)} \quad \forall f \in C_{c: \operatorname{rad}}^{1}\left(\mathbb{M}^{n}\right), \quad \forall p \in\left[\frac{2 n_{q}}{n_{q}-2}, 2^{*}\right] \cap\left[\frac{2 n_{q}}{n_{q}-2}, \infty\right),
$$

where $n_{q}$ is as in (3.41).

Also Theorem 5.2 is sharp, in the sense that on model manifolds attaining equality in (3.1) the validity of (5.2) fails for $p$ smaller than the critical exponent $2 n_{q} /\left(n_{q}-2\right)$. As a consequence, in the quasi-Euclidean case, namely when (3.1) is satisfied for some $\mu<-1$, in general we cannot guarantee more than the validity of the standard Euclidean Sobolev inequality $(2.8)$ (provided $n \geq 3$ ). 
In [19, Theorem 2.5] it is also proved that a curvature bound like (3.1) is not enough to extend either of the above results to nonradial functions. More precisely, as soon as (3.2) is satisfied for some $c, R>0$ and $\nu<0$ no inequality of the type of (5.1) can hold for nonradial functions on $\mathbb{M}^{n}$.

5.2. The radial smoothing effect for the PME on model manifolds. Theorems 5.1 and 5.2 have some interesting consequences at the level of smoothing effects for the PME problem (1.1), at least when the initial datum $u_{0}$ is radially symmetric and the manifold at hand is a model, namely it is spherically symmetric: this is crucial since it ensures that radiality is preserved along the flow, so that the Sobolev-type inequalities (5.1) or (5.2) can also be applied to the corresponding solution $u(\cdot, t)$.

Theorem 5.3 (Smoothing effect, left quasi-hyperbolic, Theorem 7.1 of [19]). Let $\mathbb{M}^{n}$ be a CartanHadamard model manifold satisfying (3.1) for some $C, R>0$ and $\mu \in(-1,0)$. Then there exists a positive constant $\mathrm{K}>0$, depending only on $n, m, \mu, C, R$, such that for every radial initial datum $u_{0} \in L^{1}\left(\mathbb{M}^{n}\right)$ the solution $u$ of (1.1) fulfills the smoothing estimate

$$
\|u(\cdot, t)\|_{\infty} \leq \mathrm{K}\left[\log \left(t\left\|u_{0}\right\|_{1}^{m-1}+e\right)\right]^{\frac{1-\mu}{(m-1)(1+\mu)}} t^{-\frac{1}{m-1}} \quad \forall t>0 .
$$

The proof of (5.3) is carried out by means of well-established techniques such as Moser iterations, interpolation inequalities and the semigroup property for (1.1). It is mostly based on ideas developed in [8, Theorem 3.1]. Here we aim at stressing the main points only, especially those in which the Sobolevtype inequalities (5.1) come into play.

Proof of Theorem 5.3. First of all, we will assume that the solution is sufficiently smooth and integrable (as well as nontrivial) in order to be able to justify our computations. We refer to Remark 5.5 below for an account of the typical techniques used to circumvent the possible lack of such properties.

Let us fix (for the moment) $t>0$ and the parameters $\alpha>0, \sigma=p / 2 \in\left(1,2^{*} / 2\right)$. Upon multiplying the differential equation in (1.1) by $u^{\alpha}$, integrating by parts and taking advantage of (5.1), we obtain:

$$
\frac{d}{d t}\|u(\cdot, t)\|_{\alpha+1}^{\alpha+1}=-\frac{4 \alpha(\alpha+1) m}{(\alpha+m)^{2}}\left\|\nabla\left(u(\cdot, t)^{\frac{\alpha+m}{2}}\right)\right\|_{2}^{2} \leq-\frac{4 \alpha(\alpha+1) m}{(\alpha+m)^{2} C_{\sigma}^{2}}\|u(\cdot, t)\|_{\sigma(\alpha+m)}^{\alpha+m},
$$

where we set

$$
\mathrm{C}_{\sigma}:=\mathrm{C} p^{\frac{1-\mu}{2(1+\mu)}}(p-2)^{\frac{\mu}{1+\mu}}=\sqrt{2} \mathrm{C} \sigma^{\frac{1-\mu}{2(1+\mu)}}(\sigma-1)^{\frac{\mu}{1+\mu}} .
$$

Note that in (5.5) we have exploited the fact that $u(\cdot, t)$ is a radial function, because so is $u_{0}$ and $\mathbb{M}^{n}$ is a model manifold. Standard interpolation in Lebesgue spaces plus the decrease of the $L^{1}\left(\mathbb{M}^{n}\right)$ norm along the evolution (see e.g. [3, Theorem 3.4]) yield

$$
\|u(\cdot, t)\|_{\alpha+1} \leq\|u(\cdot, t)\|_{\sigma(\alpha+m)}^{\frac{\sigma(\alpha+m) \alpha}{\sigma(\alpha+m)-1](\alpha+1)}}\|u(\cdot, t)\|_{1}^{\frac{\sigma(\alpha+m)-(\alpha+1)}{\sigma \sigma(\alpha+m)-1](\alpha+1)}} \leq\|u(\cdot, t)\|_{\sigma(\alpha+m)}^{\frac{\sigma(\alpha+m) \alpha}{\sigma \sigma(\alpha+m)-1](\alpha+1)}}\left\|u_{0}\right\|_{1}^{\frac{\sigma(\alpha+m)-(\alpha+1)}{[\sigma(\alpha+m)-1](\alpha+1)}} .
$$

For simplicity, let us assume $\left\|u_{0}\right\|_{1}=1$; the general case will follow by the plain observation that if $u(\cdot, t)$ is a solution to (1.1) then also $u\left(\cdot, t /\left\|u_{0}\right\|_{1}^{m-1}\right) /\left\|u_{0}\right\|_{1}$ is a solution to (1.1) with initial datum $u_{0} /\left\|u_{0}\right\|_{1}$. Hence, from (5.4) and (5.6) we infer that

$$
\frac{d}{d t}\|u(\cdot, t)\|_{\alpha+1}^{\alpha+1} \leq-\frac{4 \alpha(\alpha+1) m}{(\alpha+m)^{2} C_{\sigma}^{2}}\|u(\cdot, t)\|_{\alpha+1}^{(\alpha+1) \frac{\sigma(\alpha+m)-1}{\sigma \alpha}} .
$$

By setting $y(t):=\|u(\cdot, t)\|_{\alpha+1}^{\alpha+1}$ and integrating the above differential inequality, we end up with

$$
y(t)^{\frac{\sigma m-1}{\sigma \alpha}} \leq\left[\frac{1}{y(0)^{\frac{\sigma m-1}{\sigma \alpha}}}+\frac{4 m(\alpha+1)(\sigma m-1)}{\sigma(\alpha+m)^{2} \mathrm{C}_{\sigma}^{2}} t\right]^{-1}
$$

whence

$$
\|u(\cdot, t)\|_{\alpha+1} \leq\left[\frac{\sigma(\alpha+m)^{2} C_{\sigma}^{2}}{4 m(\alpha+1)(\sigma m-1)}\right]^{\frac{\sigma \alpha}{(\alpha+1)(\sigma m-1)}} t^{-\frac{\sigma \alpha}{(\alpha+1)(\sigma m-1)}} .
$$

It is by now a standard result that an inequality like (5.1), for a given $\sigma_{0}=p / 2 \in\left(1,2^{*} / 2\right)$, entails the validity of the smoothing estimate

$$
\|u(\cdot, t)\|_{\infty} \leq \mathrm{K} t^{-\frac{\sigma_{0}}{\left(\sigma_{0}-1\right)(\alpha+1)+\sigma_{0}(m-1)}}\left\|u_{0}\right\|_{\alpha+1}^{\frac{\left(\sigma_{0}-1\right)(\alpha+1)}{\left(\sigma_{0}-1\right)(\alpha+1)+\sigma_{0}(m-1)}}
$$

where from here on by $\mathrm{K}$ we denote a generic positive constant as in the statement that depends only on $n, m, \mu, C, R$. We stress that the proof of (5.8) follows by using (5.4) as a starting point and then setting up a Moser-type iteration: for the details we refer e.g. to [7, Corollary 5.6] or [2, Theorem 1.5]. 
The application of (5.7) at time $t / 2$ and (5.8) with time origin shifted from 0 to $t / 2$, i.e. one sees $u(\cdot, t)$ as the solution of (1.1) starting from $u(\cdot, t / 2)$ after time $t / 2$ (semigroup property), leads to

$$
\begin{aligned}
& \|u(\cdot, t)\|_{\infty} \leq \mathrm{K}\left(\frac{t}{2}\right)^{-\frac{\sigma_{0}}{\left(\sigma_{0}-1\right)(\alpha+1)+\sigma_{0}(m-1)}}\left\|u\left(\cdot, \frac{t}{2}\right)\right\|_{\alpha+1}^{\frac{\left(\sigma_{0}-1\right)(\alpha+1)}{\left(\sigma_{0}-1\right)(\alpha+1)+\sigma_{0}(m-1)}} \\
& \leq \mathrm{K}\left[\frac{\sigma(\alpha+m)^{2} \mathrm{C}_{\sigma}^{2}}{4 m(\alpha+1)(\sigma m-1)}\right]^{\frac{\sigma \alpha\left(\sigma_{0}-1\right)}{(\sigma m-1)\left[\left(\sigma_{0}-1\right)(\alpha+1)+\sigma_{0}(m-1)\right]}} t^{-\frac{\sigma_{0}(\sigma m-1)+\sigma \alpha\left(\sigma_{0}-1\right)}{\left.(\sigma m-1)\left(\sigma_{0}-1\right)(\alpha+1)+\sigma_{0}(m-1)\right]}} .
\end{aligned}
$$

Since (5.9) holds for any $\alpha>0$, by picking $\alpha=\log (t+e)$ we can rewrite it as

$$
\begin{aligned}
\|u(\cdot, t)\|_{\infty} \leq & \mathrm{K}\left(\frac{\sigma[\log (t+e)+m]^{2}}{4 m[\log (t+e)+1](\sigma m-1)}\right)^{-\frac{\sigma\left(\sigma_{0} m-1\right)}{(\sigma m-1)\left[\left(\sigma_{0}-1\right)[\log (t+e)+1]+\sigma_{0}(m-1)\right]}} \\
& \times t^{\frac{\sigma_{0}-\sigma}{(\sigma m-1)\left[\left(\sigma_{0}-1\right)[\log (t+e)+1]+\sigma_{0}(m-1)\right]}}\left(\frac{\sigma[1+m / \log (t+e)]^{2}}{4 m[1+1 / \log (t+e)](\sigma m-1)}\right)^{\frac{\sigma}{\sigma m-1}} \\
& \times \mathrm{C}_{\sigma}^{-\frac{2 \sigma\left(\sigma_{0} m-1\right)}{(\sigma m-1)\left[\left(\sigma_{0}-1\right)[\log (t+e)+1]+\sigma_{0}(m-1)\right]}}\left[\log (t+e) \mathrm{C}_{\sigma}^{2}\right]^{\frac{\sigma}{\sigma m-1}} t^{-\frac{\sigma}{\sigma m-1}} .
\end{aligned}
$$

If $\sigma$ ranges in the interval $\left(1, \sigma_{0}\right)$, then it is easy to check that the first two factors in $(5.10)$ can be bounded from above by another generic positive constant $\mathrm{K}$ that we do not relabel, whence

$$
\|u(\cdot, t)\|_{\infty} \leq \mathrm{KC}_{\sigma}^{-\frac{2 \sigma\left(\sigma_{0} m-1\right)}{(\sigma m-1)\left[\left(\sigma_{0}-1\right)[\log (t+e)+1]+\sigma_{0}(m-1)\right]}}\left[\log (t+e) \mathrm{C}_{\sigma}^{2}\right]^{\frac{\sigma}{\sigma m-1}} t^{-\frac{\sigma}{\sigma m-1}}
$$

which implies, recalling (5.5),

$$
\|u(\cdot, t)\|_{\infty} \leq \mathrm{K}(\sigma-1)^{-\frac{2 \mu \sigma\left(\sigma_{0} m-1\right)}{(1+\mu)(\sigma m-1)\left[\left(\sigma_{0}-1\right)[\log (t+e)+1]+\sigma_{0}(m-1)\right]}}\left[\log (t+e)(\sigma-1)^{\frac{2 \mu}{1+\mu}}\right]^{\frac{\sigma}{\sigma m-1}} t^{-\frac{\sigma}{\sigma m-1}} .
$$

At this stage we can also let $\sigma$ depend on $t$, upon setting

$$
\sigma=1+\frac{\sigma_{0}-1}{\log (t+e)}
$$

this yields, after some computational rearrangements,

$$
\begin{aligned}
\|u(\cdot, t)\|_{\infty} \leq & \mathrm{K}[\log (t+e)]^{\frac{2 \mu\left(\sigma_{0} m-1\right)(m-1)\left[\log (t+e)+\sigma_{0}-1\right]-(1-\mu)\left(\sigma_{0}-1\right)\left[\left(\sigma_{0}-1\right) \log (t+e)+\sigma_{0} m-1\right]}{\left[(m-1) \log (t+e)+m\left(\sigma_{0}-1\right)\right]\left[\left(\sigma_{0}-1\right) \log (t+e)+\sigma_{0} m-1\right](m-1)(1+\mu)}} \\
& \times t^{\frac{\sigma_{0}-1}{\left[(m-1) \log (t+e)+m\left(\sigma_{0}-1\right)\right](m-1)}} \\
& \times[\log (t+e)]^{\frac{1-\mu}{(m-1)(1+\mu)}} t^{-\frac{1}{m-1}}
\end{aligned}
$$

that is (5.3) when $\left\|u_{0}\right\|_{1}=1$ (up to changing again $\mathrm{K}$ ).

Note that, by virtue of Theorem 3.4 (in particular estimate (3.8) with $\nu=\mu$ ), we can assert that the time behavior of the $L^{\infty}\left(\mathbb{M}^{n}\right)$ norm estimated by (5.3) is optimal as $t \rightarrow \infty$. On the other hand, in the special case of model manifolds and when $\mu \in(-1,0)$, Theorem 5.3 provides a generalization of the $L^{\infty}\left(\mathbb{M}^{n}\right)$ bound in (3.6) for radial initial data that are merely integrable.

In agreement with the discussion in Subsection 5.1, the thesis of Theorem 5.3 is still true in the "hyperbolic" case $\mu=0$, even if $\mathbb{M}^{n}$ is not a model manifold and $u_{0}$ is not radial: this is a direct consequence of [8, Theorem 2.1] combined with [19, Theorem 5.5].

We finally address the critical case $\mu=-1$, where a Euclidean-type smoothing effect holds up to "modifying" the actual spatial dimension.

Theorem 5.4 (Smoothing effect, quasi-Euclidean critical, Theorem 7.2 of [19]). Let $\mathbb{M}^{n}$ be a CartanHadamard model manifold satisfying (3.1) for some $C, R>0$ and $\mu=-1$. Then there exists a positive constant $\mathrm{K}>0$, depending only on $n, m, C, R$, such that for every radial initial datum $u_{0} \in L^{1}\left(\mathbb{M}^{n}\right)$ the solution $u$ of (1.1) fulfills the smoothing estimate

$$
\|u(\cdot, t)\|_{\infty} \leq \mathrm{K} t^{-\frac{n_{q}}{2+n_{q}(m-1)}}\left\|u_{0}\right\|_{1}^{\frac{2}{2+n_{q}(m-1)}} \quad \forall t>0
$$

where $n_{q}$ is as in $(3.41)$.

In view of Theorem 3.12 (in particular estimate (3.43) with $\nu=\mu$ ), we can claim that also (5.11) is optimal as $t \rightarrow \infty$ in terms of behavior of the $L^{\infty}\left(\mathbb{M}^{n}\right)$ norm; moreover, in the case of model manifolds, Theorem 5.4 generalizes the $L^{\infty}\left(\mathbb{M}^{n}\right)$ bound in (3.42) for radial initial data that are merely integrable.

The proof of Theorem 5.4 is exactly the same as the one performed in Euclidean settings: note that (5.11) is nothing but (5.8) with $\sigma_{0}=n_{q} /\left(n_{q}-2\right)$ and $\alpha=0$, hence we refer again to [7, Corollary 5.6]. 
Remark 5.5 (Rigorous justification of the differential computations). The easiest way to justify the computations carried out in the proof of Theorem 5.3 consists of working with the approximate problems (2.1), up to the following additional modifications: $u_{0}$ is a smooth and compactly-supported initial datum and $u \mapsto u^{m}$ is replaced by a nondegenerate nonlinearity $u \mapsto \Phi_{\varepsilon}(u)$ (let $\varepsilon>0$ ) that satisfies $\Phi_{\varepsilon}^{\prime}(u) \geq$ $m u^{m-1}, \Phi_{\varepsilon}^{\prime}(u)>0, \Phi_{\varepsilon}(0)=0$ and converges locally uniformly to $u^{m}$ as $\varepsilon \rightarrow 0$. Indeed, let us denote as $u_{k, \varepsilon}$ the corresponding solutions, which are smooth since they solve quasilinear parabolic problems (see the standard reference [16]). The key estimate (5.4) does hold with $u \equiv u_{k, \varepsilon}$, thanks to the above bound from below on $\Phi_{\varepsilon}^{\prime}(u)$ and the Dirichlet boundary condition; this is the starting step that leads to (5.3), still with $u \equiv u_{k, \varepsilon}$. One then lets $\varepsilon \rightarrow 0, k \rightarrow \infty$ and approximates the original initial datum, observing that the smoothing estimate is stable under all these passages (for more details we refer to [9]).

\section{Future DeVElopments And OPEn PROBlems}

We discuss here some nontrivial questions that remain open after the results described in Sections 3 and 4. As we highlighted in the Introduction, diffusion on Riemannian manifolds is a very active research field, so we expect more and more projects to be carried out in the near future.

Smoothing effects in the right hyperbolic range. A very challenging question regards the validity of smoothing estimates of the type of (5.3) also for $\mu \in(0,1)$. Indeed, the upper bound (3.6) provided in Theorem 3.3 by barriers would suggest that (5.3) still holds in this range of curvature. However, it is not at all clear what kind of functional inequalities could give rise to such a smoothing estimate, as in the range $\mu \in(0,1)$ at the level of pure Sobolev-type inequalities there is no difference with respect to the case $\mu=0$, where only (2.8) and (2.9) hold. On the other hand, in the super-hyperbolic range $\mu>1$ we know that the absolute bound (4.21) is satisfied, but the proof we gave is not functional-analytic. In [8, Theorem 4.1] it was shown that any inequality of the type of $\|f\|_{p} \leq C_{p}\|\nabla f\|_{2}$ for some $p \in[1,2)$ ensures the absolute bound; yet in the same paper [8, Theorem 4.6] it was established that no such inequality can hold on Cartan-Hadamard manifolds! Hence the functional properties of super-hyperbolic manifolds should be further investigated. A good starting point in this regard could be for instance [27].

The nonradial case. As we recalled in Subsection 5.1, in general (5.1) fails when extended to radial functions. This raises a few natural questions on the behavior of solutions to (1.1) that start from nonradial data. First: is it possible to produce a nonradial initial datum $u_{0} \in L^{1}\left(\mathbb{M}^{n}\right)$ for which (5.3) is not satisfied as $t \rightarrow \infty$ ? Second: what is the largest class of (nonradial) data that comply with (5.3)? Indeed, Theorem 3.3 suggests that this kind of asymptotic behavior might be extended to initial data that, in a suitable sense, are comparable with radial functions. Third: what about the validity of (5.3) in appropriate subclasses of Cartan-Hadamard manifolds that are not models?

Asymptotic convergence results. The remarkable paper [25] was the main source of inspiration for the results presented in Section 3. One of its most important achievements is the asymptotic convergence in rescaled variables, for radial solutions to (1.1) on $\mathbb{H}^{n}$, to the "fundamental" solution (see Theorems 1.1 and 8.1 there). This is consistent with the typical asymptotics in $\mathbb{R}^{n}$, which is discussed in detail in [24, Chapter 18]; the proof exploits delicate barriers and a smart change of variables that turns the equation into a (critical) weighted Euclidean diffusion, which was studied in [20]. On the other hand, in the super-hyperbolic range $\mu>1$ we showed that the long-time behavior is driven by a separable solution, thus reproducing the same asymptotics as in bounded domains. In the quasi-hyperbolic range $\mu \in(-1,1)$ the barriers we provided in Theorems 3.3 and 3.4 suggest that convergence results in the spirit of [25] may hold, at least when the curvature does behave like $r^{2 \mu}$ as $r \rightarrow \infty$ (i.e. not only at the level of bounds). Note that in this case the existence of fundamental solutions is guaranteed by [11].

Distance and volume growth. In $\mathbb{R}^{n}$ the expansion rate of the support of a compactly-supported solution to (1.1) is precisely $\mathrm{R}(t) \approx t^{1 /[2+n(m-1)]}$ as $t \rightarrow \infty$ (see [24, Chapter 9]), in terms of distance growth. As a consequence, the support expansion in terms of volume growth is $\mathrm{V}(t) \approx t^{n /[2+n(m-1)]}$. On the other hand, estimate (3.6) shows that on quasi-hyperbolic manifolds the expansion rate of the support, still with respect to distance growth, is logarithmic: this may lead to the (wrong) conclusion that diffusion on such manifolds is slower than diffusion on $\mathbb{R}^{n}$. Indeed, a careful computation proves that at least on $\mathbb{H}^{n}$ the volume growth is $\mathrm{V}(t) \approx t^{1 /(m-1)}$, thus faster than the Euclidean one. Unfortunately, this requires an accurate estimate of the parameter $\bar{\gamma}$ appearing in (3.5), which was carried out in [25] on $\mathbb{H}^{n}$. Hence, an open problem is to identify the sharp growth rate of $\mathrm{V}(t)$ under the curvature bound (3.1) (or (3.2)), as the sole sharp results known so far are available on $\mathbb{R}^{n}$ and $\mathbb{H}^{n}$ only. We refer to [13, Section 5] for a more detailed discussion on these issues.

The linear case $\boldsymbol{m}=1$. A special mention deserves the heat equation, namely (1.1) when $m=1$. Indeed, even if it corresponds to a linear problem, the asymptotic behavior of solutions seems harder 
to describe. The very recent paper [26] establishes some interesting convergence results (with rates), in the special case of $\mathbb{H}^{n}$, towards the fundamental solution as $t \rightarrow \infty$. In order to be able to perform a similar analysis on Cartan-Hadamard manifolds, it would be of primary importance to obtain sharp pointwise bounds on the heat kernel (i.e. the fundamental solution) of $\mathbb{M}^{n}$ under the curvature bounds (3.1) or (3.2). On the hyperbolic space the heat kernel is explicitly known (see again [26]), and it has a nontrivial structure. Surprisingly, still in [26] it is shown that for nonradial data in general the asymptotic convergence to the fundamental solution fails, at least in dimension $n=3$. This highlights that such a diffusion phenomenon is intrinsically complicated. For related issues, see also [12, Section 10].

Conflict of interest. The author states that there is no conflict of interest.

\section{REFERENCES}

[1] Ph. Bénilan and M.G. Crandall, The continuous dependence on $\varphi$ of solutions of $u_{t}-\Delta \varphi(u)=0$, Indiana Univ. Math. J. 30 (1981), 161-177.

[2] M. Bonforte and G. Grillo, Asymptotics of the porous media equation via Sobolev inequalities, J. Funct. Anal. 225 (2005), 33-62.

[3] M. Bonforte, G. Grillo and J.L. Vázquez, Fast diffusion flow on manifolds of nonpositive curvature, J. Evol. Equ. 8 (2008), 99-128.

[4] H. Brezis and S. Kamin, Sublinear elliptic equations in $\mathbb{R}^{n}$, Manuscripta Math. 74 (1992), 87-106.

[5] M. Ghomi and J. Spruck, Total curvature and the isoperimetric inequality in Cartan-Hadamard manifolds, preprint arXiv: https://arxiv.org/abs/1908.09814.

[6] A. Grigor'yan, Analytic and geometric background of recurrence and non-explosion of the Brownian motion on Riemannian manifolds, Bull. Amer. Math. Soc. 36 (1999), 135-249.

[7] G. Grillo and M. Muratori, Sharp short and long time $L^{\infty}$ bounds for solutions to porous media equations with homogeneous Neumann boundary conditions, J. Differential Equations 254 (2013), 2261-2288.

[8] G. Grillo and M. Muratori, Smoothing effects for the porous medium equation on Cartan-Hadamard manifolds, Nonlinear Anal. 131 (2016), 346-362.

[9] G. Grillo, M. Muratori and M.M. Porzio, Porous media equations with two weights: smoothing and decay properties of energy solutions via Poincaré inequalities, Discrete Contin. Dyn. Syst. 33 (2013), 3599-3640.

[10] G. Grillo, M. Muratori and F. Punzo, On the asymptotic behaviour of solutions to the fractional porous medium equation with variable density, Discrete Contin. Dyn. Syst. 35 (2015), 5927-5962.

[11] G. Grillo, M. Muratori and F. Punzo, The porous medium equation with measure data on negatively curved Riemannian manifolds, J. Eur. Math. Soc. (JEMS) 20 (2018), 2769-2812.

[12] G. Grillo, M. Muratori and J.L. Vázquez, The porous medium equation on Riemannian manifolds with negative curvature. The large-time behaviour, Adv. Math. 314 (2017), 328-377.

[13] G. Grillo, M. Muratori and J.L. Vázquez, The porous medium equation on Riemannian manifolds with negative curvature: the superquadratic case, Math. Ann. 373 (2019), 119-153.

[14] E. Hebey, "Nonlinear Analysis on Manifolds: Sobolev Spaces and Inequalities". Courant Lecture Notes in Mathematics, 5. New York University, Courant Institute of Mathematical Sciences, New York; American Mathematical Society, Providence, RI, 1999.

[15] S. Kamin, G. Reyes and J.L. Vázquez, Long time behavior for the inhomogeneous PME in a medium with rapidly decaying density, Discrete Contin. Dyn. Syst. 26 (2010), 521-549.

[16] O.A. Ladyženskaja, V.A. Solonnikov and N.N. Ural'ceva, "Linear and Quasilinear Equations of Parabolic Type". American Mathematical Society, Providence, RI, 1968.

[17] J.M. Lee, "Riemannian Manifolds. An Introduction to Curvature". Graduate Texts in Mathematics, 176. SpringerVerlag, New York, 1997.

[18] H.P. McKean, An upper bound to the spectrum of $\Delta$ on a manifold of negative curvature, J. Differential Geometry 4 (1970), 359-366.

[19] M. Muratori and A. Roncoroni, Sobolev-type inequalities on Cartan-Hadamard manifolds under curvature bounds, preprint arXiv: https://arxiv.org/abs/1805.02726.

[20] S. Nieto and G. Reyes, Asymptotic behavior of the solutions of the inhomogeneous porous medium equation with critical vanishing density, Commun. Pure Appl. Anal. 12 (2013), 1123-1139.

[21] G. Reyes and J.L. Vázquez, Long time behavior for the inhomogeneous PME in a medium with slowly decaying density, Commun. Pure Appl. Anal. 8 (2009), 493-508.

[22] J.L. Vázquez, The Dirichlet problem for the porous medium equation in bounded domains. Asymptotic behavior, Monatsh. Math. 142 (2004), 81-111.

[23] J.L. Vázquez, "Smoothing and Decay Estimates for Nonlinear Diffusion Equations. Equations of Porous Medium Type". Oxford University Press, Oxford, 2006.

[24] J.L. Vázquez, "The Porous Medium Equation. Mathematical Theory". The Clarendon Press, Oxford University Press, Oxford, 2007.

[25] J.L. Vázquez, Fundamental solution and long time behavior of the porous medium equation in hyperbolic space, J. Math. Pures Appl. 104 (2015), 454-484.

[26] J.L. Vázquez, Asymptotic behaviour for the heat equation in hyperbolic space, preprint arXiv: https://arxiv.org/ abs/1811.09034.

[27] F.-Y. Wang, Intrinsic ultracontractivity on Riemannian manifolds with infinite volume measures, Sci. China Math. 53 (2010), 895-904. 
Matteo Muratori, Dipartimento di Matematica, Politecnico di Milano, Piazza Leonardo da Vinci 32, 20133 Milano, Italy

Email address: matteo.muratori@polimi.it 